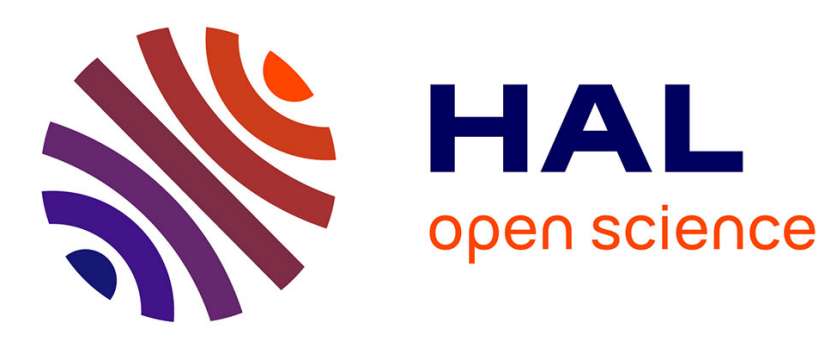

\title{
Vibrational Spectra Obtained from High Quality Potential Energy Surfaces Spanned by Low Level Normal Coordinates - Application to CHFClI and CDFClI
}

\author{
Guntram Rauhut, Peter Schwerdtfeger, Sandra Heislbetz
}

\section{To cite this version:}

Guntram Rauhut, Peter Schwerdtfeger, Sandra Heislbetz. Vibrational Spectra Obtained from High Quality Potential Energy Surfaces Spanned by Low Level Normal Coordinates - Application to CHFClI and CDFClI. Molecular Physics, 2007, 105 (10), pp.1385-1394. 10.1080/00268970701348741 . hal00513095

\section{HAL Id: hal-00513095 \\ https://hal.science/hal-00513095}

Submitted on 1 Sep 2010

HAL is a multi-disciplinary open access archive for the deposit and dissemination of scientific research documents, whether they are published or not. The documents may come from teaching and research institutions in France or abroad, or from public or private research centers.
L'archive ouverte pluridisciplinaire HAL, est destinée au dépôt et à la diffusion de documents scientifiques de niveau recherche, publiés ou non, émanant des établissements d'enseignement et de recherche français ou étrangers, des laboratoires publics ou privés. 


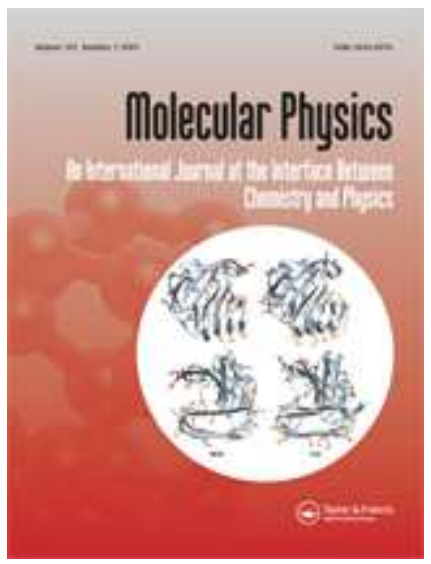

\section{Vibrational Spectra Obtained from High Quality Potential Energy Surfaces Spanned by Low Level Normal Coordinates - Application to CHFCII and CDFCII}

\begin{tabular}{|c|c|}
\hline Journal: & Molecular Physics \\
\hline Manuscript ID: & TMPH-2007-0049.R2 \\
\hline Manuscript Type: & Full Paper \\
\hline $\begin{array}{l}\text { Date Submitted by the } \\
\text { Author: }\end{array}$ & 19-Mar-2007 \\
\hline Complete List of Authors: & $\begin{array}{l}\text { Rauhut, Guntram; Universitity of Stuttgart, Institute of Theoretical } \\
\text { Chemistry } \\
\text { Schwerdtfeger, Peter; Massey University, Centre of Theoretical } \\
\text { Chemistry and Physics } \\
\text { Heislbetz, Sandra; University of Stuttgart, Institute of Theoretical } \\
\text { Chemistry }\end{array}$ \\
\hline Keywords: & VSCF calculations, CHFCII \\
\hline \multicolumn{2}{|c|}{$\begin{array}{l}\text { Note: The following files were submitted by the author for peer review, but cannot be converted } \\
\text { to PDF. You must view these files (e.g. movies) online. }\end{array}$} \\
\hline TMPH-2007-0049.tex & \\
\hline
\end{tabular}

\section{S ScholaroNE" \\ Manuscript Central}




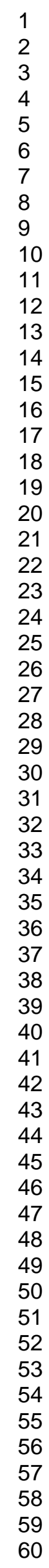

URL: http://mc.manuscriptcentral.com/tandf/tmph 


\title{
Vibrational Spectra Obtained from High Quality Potential Energy Surfaces Spanned by Low Level Normal Coordinates - Application to CHFCII and CDFClI
}

\author{
Sandra Heislbetz ${ }^{1}$, Peter Schwerdtfeger ${ }^{2}$, and Guntram Rauhut*,1 \\ 1 Institut für Theoretische Chemie, Universität Stuttgart, Pfaffenwaldring 55, 70569 \\ Stuttgart, Germany. \\ ${ }^{2}$ Centre of Theoretical Chemistry and Physics (CTCP), Bldg. 44, Institute of Fundamen- \\ tal Sciences, Massey University (Auckland Campus), Private Bag 102904, North Shore \\ MSC, Auckland, New Zealand.
}

\section{Abstract}

The impact of approximate normal coordinates for spanning high level potential energy surfaces on vibrational frequencies is studied within the framework of vibrational SCF and configuration interaction calculations (VCI). The use of low level normal coordinates avoids expensive geometry optimizations and harmonic frequency calculations and thus allows for a significant reduction in CPU time. Benchmark calculations are provided for a set of molecules ranging from 5 to 7 atoms. An application to CHFClI and CDFCII shows that this approximation still allows for very accurate results. These molecules are of particular interest for studying parity violation effects.

\footnotetext{
*To whom correspondence should be addressed: rauhut@theochem.uni-stuttgart.de
} 


\section{Introduction}

The calculation of accurate vibrational spectra is a time consuming task. At reliable computational levels, e.g. CCSD (T)/aug-cc-p(C)VTZ and beyond, such calculations may require several weeks of CPU time even for small molecules and within the harmonic approximation. Consequently, most computational studies on vibrational spectra are based on density functional theory rather than wave function based $a b$ initio methods [1]. In most approaches for calculating anharmonic vibrational spectra, the computation of a reliable harmonic spectrum is a necessary prerequisite. Within perturbational approaches, the anharmonic corrections are simply added to the harmonic values [2-4], while for variational vibrational SCF calculations (VSCF) the normal modes of the harmonic calculation are often used within the expansion of the potential [5-7]. In perturbational calculations it appears to be common practice to compute the anharmonic corrections at a lower computational level than the harmonic frequencies $[1,3,4]$. In analogy to this, the expansion of the potential in terms of many mode representations can be performed at different computational levels as well, i.e. important one-mode terms (1D) need to be computed at the highest level, while for the less important three-mode or four-mode coupling terms (3D and 4D) lower theoretical levels appear to be sufficient $[8,9]$. The impact of the approximation was found to be very little once the different computational levels have been chosen carefully. For example, a 1D:CCSD(T)-2D:MP4(SDQ)-3D:MP2 multi-level scheme appears to be quite successful within the calculation of anharmonic vibrational spectra $[8,10]$. As the multidimensional potential calculated by a multi-level scheme does not refer exactly to the normal modes used for spanning the potential, the question arises if normal modes at lower computational levels can be used whithout introducing significant errors. Of course, this is not an approach for very accurate calculations (e.g. Ref. 11,12), but if the errors remain reasonably small the achieved accuracy may be sufficient for many applications while the overall CPU-time can be significantly reduced. Such a method would be appealing for two reasons: (1) low level calculations like MP2 or DFT are much faster than coupled-cluster calculations with a perturbational treatment of triple excitations. (2) For many low level methods analytical first and/or second order derivatives are available, while for the more sophisticated methods, e.g. density fitting $\operatorname{CCSD}(\mathrm{T})$, second derivatives need to be computed by twofold numerical differentiation which scales quadratically with respect to the degrees of freedom rather than linearly. 
Here we present a systematic study on the impact of different normal modes used for spanning multi-level potential energy surfaces on anharmonic vibrational frequencies. All calculations are based on configuration-selective vibrational CI calculations (cs-VCI) as will be described in detail below. In section 4 we present calculations for the fundamental modes of CHFClI and CDFClI which will be compared with the most recent experimental results of Soulard et al. [13]. These molecules are of particular interest as they are potential candidates for significant parity violation effects $[14,15]$. In addition, this study extends our work on chiral methane derivatives (see Ref. 10).

\section{Approximate normal coordinates}

\subsection{Computational details}

A small set of molecules ranging from 4 to 7 atoms has been used for studying the impact of the normal modes obtained from different electronic structure levels on the fundamental modes obtained from multi-level VCI calculations. This test set comprises formaldehyde [16], phosphorus trichloride [17], dibromomethane [18, 19], trans-difluoroethylene [20], vinyl chloride [21] and 1,2,5-oxadiazole [8,22]. Using a cc-pVTZ basis set throughout [23], all structures were optimized at the MP2, MP4(SDQ) and CCSD(T) levels of theory. The innermost 10 electrons of bromine (in dibromomethane) were approximately treated by a Stuttgart small core relativistic effective core potential ECP10MDF with a correlation consistent basis set adjusted by Peterson et al. [25]. Harmonic frequencies and thus the normal coordinates were computed at the same electronic structure levels. These coordinates were used for spanning multi-level 1D:CCSD(T)-2D:MP4(SDQ)-3D:MP2 potentials (see equation 1) which were applied in the subsequent variational VSCF and VCI calculations based on the Watson Hamiltonian [26]. 
In all calculations presented here, this series has been truncated after the 3D terms, which was found to be sufficient for most applications. The correction due the 4D terms, which are fully implemented in our code, is very small. The difference potentials $V_{i}\left(q_{i}\right), V_{i j}\left(q_{i}, q_{j}\right)$ etc. are given as

$$
\begin{aligned}
V_{i}\left(q_{i}\right) & =V_{i}^{0}\left(q_{i}\right)-V_{0} \\
V_{i j}\left(q_{i}, q_{j}\right) & =V_{i j}^{0}\left(q_{i}, q_{j}\right)-\sum_{r \in\{i, j\}} V_{r}\left(q_{r}\right)-V_{0}
\end{aligned}
$$

and likewise for all other terms (3D and 4D). All quantities with the superscript 0 denote the total energy as obtained from the electronic structure calculations at distorted nuclear configurations. $V_{0}$ represents the total energy at the equilibrium geometry. In order to determine the incremental contributions in such a scheme correctly, one would in principle need to compute the one-mode (1D) contributions at all three levels and the 2D terms at the MP4(SDQ) and MP2 levels. This repeated calculation of the $1 \mathrm{D}$ and $2 \mathrm{D}$ grid points is necessary in order to generate consistent difference potentials which obey the conditions:

$$
V_{i j}\left(q_{i}, q_{j}=0\right)=V_{i j}\left(q_{i}=0, q_{j}\right)=0
$$

However, as in the MoLPRo suite of ab initio programs [27] the MP2 energy is a byproduct of the $\operatorname{CCSD}(\mathrm{T})$ and $\mathrm{MP} 4(\mathrm{SDQ})$ computational schemes, in practice only the $1 \mathrm{D}$ terms need to be computed twice, i.e. at the $\operatorname{CCSD}(\mathrm{T})$ and MP4(SDQ) levels. Consequently, the additional computational effort is rather limited. The multi-level scheme has not been changed during this study. We explicitly note here, that this computational scheme usually is not sufficient to ensure an accuracy which results in excellent agreement with experimental data. Usually larger basis sets including diffuse functions [28], core correlation effects, and spin-orbit couplings appear to be important $[8,12]$. However, the computational scheme used here is accurate enough in order to exclude substantial 
artifacts and to allow for a comparison with experimental data. As reported in detail elsewhere [9] the potential energy surfaces of all molecules within this study were generated by a fully automated and parallelized algorithm which is based on an iterative interpolation technique and a prescreening scheme for the 3D contributions. Each difference potential is represented by 16 grid points in each dimension. These fine grids are obtained from one-dimensional polynomial interpolations based on an increasing number of coarse grid points. Within the VSCF approach single-mode vibrational wave functions (modals) were generated from 16 distributed Gaussians using a collocation algorithm [29]. Vibrational correlation effects were accounted for by a configuration-selective VCI approach, i.e. cs-VCI, including configurations with at most four different excited modals (quadruple excitations) while restricting the maximum excitation level within one modal to the fourth root. In addition, the overall excitation level was restricted to seven. From these initially generated configurations a subset has been selected for the final VCI calculations using a recently developed configuration selection scheme. All these algorithms are implemented in a development version of the MOLPRO suite of ab initio programs [27].

\section{$3.2 \quad$ Results}

Tables I - V show the results for the fundamental modes of the test set molecules except for 1,2,5-oxadiazole. In all these tables, the frequencies based on potentials spanned by $\operatorname{CCSD}(\mathrm{T}) / \mathrm{cc}-\mathrm{pVTZ}$ normal coordinates were taken as the reference (last three columns). The harmonic MP2 and MP4(SDQ) frequencies usually lead to a strong systematic overestimation with respect to the $\operatorname{CCSD}(\mathrm{T})$ results. The mean absolute deviation for MP2 is $15.7 \mathrm{~cm}^{-1}$ with maximum deviations as large as $47.4 \mathrm{~cm}^{-1}$. Based on these results one is also tempted to expect rather significant deviations between the MP2 and CCSD(T) normal coordinates. However, calculation of the dot products of the normal coordinate displacement vectors obtained at different levels of electronic structure theory shows that this is not necessarily the case. As a result, the VSCF and cs-VCI frequencies obtained from potentials spanned by low level (i.e. MP2 or MP4(SDQ)) calculations usually closely resemble those obtained from $\operatorname{CCSD}(\mathrm{T})$ normal coordinates. For VSCF calculations, the mean absolute deviation is as low as $0.3 \mathrm{~cm}^{-1}$ for MP2 and $0.5 \mathrm{~cm}^{-1}$ for MP4(SDQ), respectively. For cs-VCI calculations this deviation is slightly larger: $0.5 \mathrm{~cm}^{-1}$ for MP2 
and $0.6 \mathrm{~cm}^{-1}$ for MP4(SDQ) normal coordinates. The enhanced sensitivity of the VCI results mirrors the observation in electronic structure theory that correlation methods are more sensitive to the basis set size and integral accuracy than Hartree-Fock calculations. Maximum deviations scatter in the range between 0.3 and $3.0 \mathrm{~cm}^{-1}$, the latter observed for the $1 a^{\prime}$ mode of vinyl chloride (MP2, Table IV). At the MP4(SDQ) level the maximum deviation decreases to $2.2 \mathrm{~cm}^{-1}$. These errors are far below the intrinsic error of the electronic structure levels. Usually, core correlation effects or spin-orbit couplings can be as large as $10 \mathrm{~cm}^{-1}$. The same holds true for increasing the basis set from triple- $\zeta$ to quadruple- $\zeta$ quality. Consequently, the approximation introduced appears to be save if no higher accuracy is desired.

The substitution of $\operatorname{CCSD}(\mathrm{T})$ normal coordinates by low level coordinates is based on the assumption that both sets strongly resemble each other. The question arises, what happens if this cannot be guaranteed? For this very reason we have included 1,2,5oxadiazole into the test set. This is a molecule which is poorly represented by MP2 and even MP4(SDQ) calculations. For example, the CN bond lengths differ from the CCSD(T) bond length by $0.016 \AA$ at the MP2 level and by $0.010 \AA$ at the MP4(SDQ) level, respectively. Likewise, the CC bond length deviates at the MP2 level by $0.023 \AA$ from the coupled-cluster value. Dot products between the MP2 and the CCSD(T) normal coordinate displacement vectors can be as low as $0.81\left(6 a_{1}\right)$. Consequently, larger deviations than for the other test molecules must be anticipated in this case. VCI results for this molecule are provided in Table VI. As expected, a mean absolute deviation of $2.7 \mathrm{~cm}^{-1}$ at the MP2 level is far above the value for the other molecules and a maximum deviation of $9.8 \mathrm{~cm}^{-1}$ for the $3 a_{1}$ mode is not acceptable. This prompted us to use methods for calculating normal coordinates, which are not included in the multi-level approximation of the potential, but simply provide a reliable approximation to the coupled-cluster coordinates. Two methods appear to be particularly promising in that respect: (1) density functional methods which are known for a proper representation of vibrational frequencies and (2) the QCISD(T) method, which can be considered a truncated coupled-cluster variant [30]. QCISD $(\mathrm{T})$ calculations are not significantly faster than $\operatorname{CCSD}(\mathrm{T})$ calculations, but for the former one analytical gradients are available in MoLPRo which avoids the twofold numerical differentiation [24]. These results are also included in Table VI. Both 
methods yield results which are in excellent agreement with the coupled-cluster results. Furthermore, the deviations introduced by this approximation are significantly smaller than the error obtained from the limited basis set or the neglect of core-correlation effects. Consequently, it occurs that even problematic molecules can be handled within this approximation and thus expensive geometry optimizations and harmonic frequency calculations at the $\operatorname{CCSD}(\mathrm{T})$ appear not to be necessary for most systems.

The saving in CPU-time due to the introduced approximation can be quite substantial. As outlined above, the savings are due to 2 reasons: (1) the use of analytical first derivatives instead of a twofold numerical differentiation leads to a reduction from quadratic to linear scaling within the generation of the normal coordinates. (2) The formal scaling with respect to the molecular size of the low level correlation methods $\left(O\left(N^{5}\right)\right.$ for MP2 and $O\left(N^{4}\right)$ for DFT) is significantly better than for $\operatorname{CCSD}(\mathrm{T})$, i.e. $O\left(N^{7}\right)$. For the molecules of the test set this leads to speed-ups in the generation of the normal modes up to a factor of about 40. Consequently, the overall calculation time can be reduced significantly without introducing substantial errors.

\section{The vibrational spectra of CHFCII and CDFClI}

\subsection{Computational details}

The structure of CHFClI has been optimized at the MP2 and CCSD(T) levels in combination with aug-cc-pVTZ and aug-cc-pVQZ basis sets. Core correlation effects have not been included and the influence of the innermost 28 electrons of iodine was simulated by a Stuttgart small core relativistic effective core potential (ECP28MDF) with correlation consistent basis sets adjusted by Peterson [25]. Harmonic frequencies were computed for both isotopomers at the MP2/aug-cc-pVTZ, MP2/aug-cc-pVQZ and CCSD(T)/aug-ccpVTZ levels. While first analytical derivatives could be used for the MP2 calculation, the calculation of the $\operatorname{CCSD}(\mathrm{T})$ harmonic frequencies required a twofold numerical differentiation step. 
Potential energy surfaces were spanned either by normal coordinates obtained from MP2/augcc-pVTZ or CCSD(T)/aug-cc-pVTZ calculations and were generated from a multi-level 1D:CCSD(T)-2D:MP4(SDQ)-3D:MP2 scheme in combination with an aug-cc-pVTZ basis set [9]. In total 2636 grid points (1D: 88, 2D: 796 and 3D: 1752) had to be computed at the different computational levels. The frozen core approximation has been used throughout. Calculation of the potential on a 2 processor dual core AMD Opteron 285 (2.6 GHz) takes slightly less than 5 days. Within the VSCF and VCI calculations the same conditions have been used as discussed in detail above for the test molecules. In the VCI calculations about 500 configurations (selected from 7675 initially generated configurations) were needed on average in order to yield converged results.

\subsection{Results}

Results for all internal coordinates of the equilibrium structure are provided in Table VII. The same trends are observed as in our previous study on CHFClBr [10]: In comparison to the MP2 results, CCSD(T) leads to slightly larger bond lengths. We address this general trend to the overestimation of correlation effects in MP2 calculations. Primarily this affects the $\mathrm{CCl}$ and $\mathrm{CI}$ bonds lengths. However, the overall agreement between the MP2 and CCSD(T) coordinates is excellent, in particular for the larger aug-cc-pVQZ basis set. Moreover, based on the $\operatorname{CCSD}(\mathrm{T}) /$ aug-cc-pVnZ, $n=2-4$, equilibrium bond lengths we have also computed the $r_{e}$ bond lengths for the complete basis set (CBS) limit using the three-parameter extrapolation formula of Peterson et al. [31] (denoted CBS-3) and the two-parameter equation (CBS-2) of Helgaker et al. [32,33]. These bond lengths must be considered the most accurate values. The two different approaches used for calculating the basis set limit provide an error bar of about $\pm 0.0005 \AA$. Surprisingly, the CBS-3 value for the $\mathrm{CH}$ bond is larger than the CCSD(T)/aug-cc-pVQZ result. This may originate from a rather short CCSD $(\mathrm{T}) /$ aug-cc-pVTZ value which is already very close to the CCSD(T)/aug-cc-pVQZ bond length. However, the uncertainty of the CBS results is larger than the elongation with respect to the $\operatorname{CCSD}(\mathrm{T}) /$ aug-cc-pVQZ bond length. The CBS values indicate that except for the $\mathrm{CH}$ bond even the aug-cc-pVQZ results are far from being converged. The largest correction is found for the $\mathrm{CCl}$ bond length, but it is well known that for 2nd-row atoms the basis set exprapolation based on aug-cc-pVnZ 


\section{Molecular Physics}

bases may lead to nonnegligible errors [34] and thus we expect the CBS value for the $\mathrm{CCl}$ bond length to be less accurate than for the other bonds. In order to account for vibrational effects on the structural parameters, anharmonicity corrections were computed from the expectation value using the aug-cc-pVTZ multi-level VCI wavefunction of the vibrational ground-state. These corrections were applied to both, the MP2 and CCSD(T) equilibrium structures which refer to the aug-cc-pVTZ basis set. Results are shown in Table VIII. Here, the most important result is that the MP2 and CCSD(T) values results coincide due to the vibrational corrections - which of course is expected as long as the wave functions of the vibrational ground state are very similar. While these corrections are rather large in the MP2 case, they are significantly smaller for the CCSD(T) calculation. In other words, without performing a $\operatorname{CCSD}(\mathrm{T})$ geometry optimization, one is able to reproduce the coupled-cluster geometrical parameters - but of course in a more economic way. To the best of our knowledge experimental geometrical parameters are not available for this molecule. However, Soulard et al. [13] provide rotational constants for the $\mathrm{CH}^{35} \mathrm{ClFI}$ and $\mathrm{CH}^{37} \mathrm{FClI}$ isotopomers corresponding to vibrationally averaged geometries. Hence, we computed these constants for the equilibrium and the vibrationally averaged structures (cf. Table IX). The rotational constants were not computed via the expectation value of the eigenvalues of the moment of inertia tensor for the vibrational ground state but rather from the moment of inertia tensor of the vibrationally averaged structure. With respect to the equilibrium values still a considerable basis set dependence can be seen and for the A constant the CBS values still differ considerably from the augcc-pVQZ result. The two different methods for calculating the complete basis set limit again provide a measure for the uncertainty within these values. Empirical corrections to the $\mathrm{CCl}$ and $\mathrm{CI}$ bond lengths would yield slightly shorter bonds and thus larger rotational constants [35]. As our calculations do not include core-correlation effects, which - in line with the empirical corrections - usually lead to shorter bonds, we consider the CBS values provided in Table IX being slightly too low. However, as the experimental values and the computed values at the equilibrium geometry refer to different structures $\left(r_{e}\right.$ instead of $r_{z}$ ), this is not a fair comparison (although it is frequently done in the literature). Consequently, the only meaningful comparison is between the experimental values and the vibrationally averaged structure. The computed vibrationally averaged values underestimate the experimental values, indicating that the CCSD $(\mathrm{T}) /$ aug-cc-pVTZ structure is not yet fully converged. 
The harmonic frequencies and their corresponding intensities (obtained from analytical dipole moment derivatives) are listed in Tables X and XI. While basis sets (from aug-ccpVTZ to aug-cc-pVQZ) appear to be rather small (the maximum deviation $\Delta \omega_{\max }$ at the MP2 level is $5.2 \mathrm{~cm}^{-1}$ ), differences between MP2 and $\operatorname{CCSD}(\mathrm{T})$ are still quite substantial $\left(\Delta \omega_{\max }=24.3 \mathrm{~cm}^{-1}\right)$. While a direct comparison of the harmonic frequencies of CHFClI with the experimental results of Soulard et al. [13] (cf. Table XII) remains unsatisfying, it is interesting to note, that the lowest three vibrations $\left(\omega_{7}-\omega_{9}\right)$ are properly represented by both, the harmonic MP2 and the harmonic $\operatorname{CCSD}(\mathrm{T})$ calculations. Consequently, these modes appear to be less sensitive to electron correlation effects and are fairly harmonic in nature, while anharmonic corrections are important for all other modes. The overall agreement of the fundamental VCI modes (cf. Table XII) with the experimental values is excellent. The mean absolute deviation is $4.6 \mathrm{~cm}^{-1}$ while the maximum deviation is about twice as large $\left(8.3 \mathrm{~cm}^{-1}\right)$. The approximation introduced above for reducing the computational effort within the calculation of the normal modes essentially leads to negligible deviations for both isotopomers (mean absolute deviation: $0.3 \mathrm{~cm}^{-1}$, maximum deviation ( $\mathrm{CCl}$ stretching mode): $1.1 \mathrm{~cm}^{-1}$ ). This result is not unexpected as the MP2 structural parameters closely resemble the $\operatorname{CCSD}(\mathrm{T})$ results (see above). On the other hand, the savings in CPU-time amount to about 20 days for this system, when running the harmonic frequency calculations on just one processor. No experimental values are available for CDFClI in the literature. Therefore, the data compiled here must be considered a prediction. However, we do not anticipate any larger errors for this isotopomer than for CHFClI. On the contrary, in analogy to our previous study on CHFClBr and $\mathrm{CDFClBr}$ we expect slightly smaller deviations with respect to experimentally observed values.

\section{Summary and Conclusions}

It could be shown that for the calculation of accurate vibrational spectra, it is not necessary to optimize the structural parameters of molecules at the highest levels of electronic structure theory. As long as the structural parameters of a molecule obtained by a low level 
method closely resemble those of high level methods, e.g. CCSD(T)/aug-cc-pVTZ (typically deviations should be less than 1\%), it appears that despite considerable differences in the harmonic frequencies the impact of the low level normal coordinates on the VCI frequencies is rather small - a very few wavenumbers only. Modern density functionals, which are known to be particular successful in the reproduction of structural parameters and harmonic vibrational spectra, appear to be the most promising low-level approaches for the generation of approximate normal coordinates. Even for small molecules, this may result in significant time savings. For the systems presented here, the fairly accurate multi-level VCI calculations (i.e. surface generation plus a vibrational CI calculation) usually takes about as long as a corresponding high-level harmonic frequency calculation. However, it must be admitted that this ratio holds only true as long as a twofold numerical differentiation at the high level cannot be avoided.

The structure and vibrational frequencies of CHFCII and CDFClI have been studied by accurate calculations up to the $\operatorname{CCSD}(\mathrm{T}) /$ aug-cc-pVQZ level. Fundamental frequencies obtained from multi-level vibrational CI calculations are in excellent agreement with recent experimental results of Soulard et al. [13] for CHFClI. Maximum deviations are as low as $8.3 \mathrm{~cm}^{-1}$. In addition to that we present here predictions for the deuterated isotopomer.

\section{Acknowledgements}

This work was performed within the Collaborative Research Centre SFB 706 (Selective Catalytic Oxidations Using Molecular Oxygen; Stuttgart) and funded by the German Research Foundation. The authors thank Prof. P. Botschwina for helpful discussions and Dr. J. Crassous for a copy of the Raman spectrum of CHFClI. P. Schwerdtfeger acknowledges financial support from Marsden fund and the ISAT Linkage fund (Wellington). 


\section{References}

[1] A.D. Boese and J.M.L. Martin, J. Phys. Chem. A 108, 3085 (2004).

[2] D.A. Clabo Jr., W.D. Allen, R.B. Remington, Y. Yamaguchi, and H.F. Schaefer, Chem. Phys. 123, 187 (1988).

[3] H. Bürger, R. Kuna, S. Ma, J. Breidung, and W. Thiel, J. Chem. Phys. 101, 1 (1994).

[4] H. Bürger, S. Ma, J. Breidung, and W. Thiel, J. Chem. Phys. 104, 4945 (1996).

[5] J.M. Bowman, Acc. Chem. Res. 19, 202 (1986).

[6] J.M. Bowman, J. Chem. Phys. 68, 608 (1978).

[7] R. Gerber and M. Ratner, Adv. Chem. Phys. 70, 97 (1988).

[8] K. Pflüger, M. Paulus, S. Jagiella, T. Burkert, and G. Rauhut, Theor. Chem. Acc. 114, 327 (2005).

[9] G. Rauhut, J. Chem. Phys. 121, 9313 (2004).

[10] G. Rauhut, V. Barone, and P. Schwerdtfeger, J. Chem. Phys. 125, 054308 (2006).

[11] S.N. Yurchenko, J. Breidung and W. Thiel, Theor. Chem. Acc. 114, 333 (2005).

[12] J. Koput and K.A. Peterson, J. Chem. Phys. 125, 044306 (2006). 
[13] P. Soulard, P. Asselin, A. Cuisset, J.R.A. Moreno, T.R. Huet, D. Petitprez, J. Demaison, T.B. Freedman, X. Cao, L.A. Nafie, and J. Crassous, Phys. Chem. Chem. Phys. 8, 79 (2006).

[14] P. Schwerdtfeger, J.K. Laerdahl, and C. Chardonnet, Phys. Rev. A 65, 042508 (2002).

[15] P. Schwerdtfeger, T. Saue, J.N.P. van Stralen, and L. Visscher, Phys. Rev. A 71, 012103 (2005).

[16] D.E. Reisner, R.W. Field, J.L. Kinsey, and H.-L. Dai, J. Chem. Phys. 80, 5968 (1984).

[17] A. Ruoff, H. Burger, S. Biedermann, and J. Cichon, Spectrochim. Acta Part A 28, 953 (1972).

[18] R.S. Dennen, E.A. Piotrowski, and F.F. Cleveland, J. Chem. Phys. 49, 4385 (1968).

[19] C. Lugez, A. Shriver, L. Shriver-Mazzuoli, E. Lasson, and C.J. Nielsen, J. Chem. Phys. 97, 11617 (1993).

[20] N.C. Craig, K.L. Petersen, and D.C. McKean, J. Phys. Chem. A 106, 6358 (2002).

[21] W.A. Herrebout and B.J. van der Veken, J. Chem. Soc., Faraday Trans. 93, 3453 (1997).

[22] D.H. Christensen and O.F. Nielsen, J. Mol. Spectrosc. 24, 477 (1967).

[23] T.H. Dunning, J. Chem. Phys. 90, 1007 (1989).

[24] G. Rauhut and H.J. Werner, Phys. Chem. Chem. Phys. 3, 4853 (2001).

[25] K.A. Peterson, D. Figgen, E. Goll, H. Stoll, and M. Dolg, J. Chem. Phys. 119, 11113 (2003). 
[26] J.K.G. Watson, Mol. Phys. 15, 479 (1968).

[27] Molpro, Development Version 2006.2, is a package of ab initio programs written by H.-J. Werner and P. J. Knowles, with contributions from R. D. Amos, A. Bernhardsson, A. Berning, P. Celani, D. L. Cooper, M. J. O. Deegan, A. J. Dobbyn, F. Eckert, C. Hampel, G. Hetzer, T. Korona, R. Lindh, A. W. Lloyd, F. R. Manby, W. Meyer, S. J. McNicholas, M. E. Mura, A. Nicklass, P. Palmieri, K. Peterson, P. Pulay, G. Rauhut, M. Schütz, U. Schumann, H. Stoll, A. J. Stone, R. Tarroni, and T. Thorsteinsson., (see http://www.molpro.net/).

[28] R.A. Kendall, T.H. Dunning, and R.J. Harrison, J. Chem. Phys. 96, 6769 (1992).

[29] W. Yang and A.C. Peet, Chem. Phys. Lett. 153, 98 (1988).

[30] J.A. Pople, M. Head-Gordon, and K. Raghavachari, J. Chem. Phys. 87, 5968 (1987).

[31] K.A. Peterson, D.E. Woon, and T.H. Dunning, J. Chem. Phys. 100, 7410 (1994).

[32] T. Helgaker, W. Klopper, H. Koch, and J. Noga, J. Chem. Phys. 106, 9639 (1997).

[33] A. Halkier, T. Helgaker, P. Jørgensen, W. Klopper, H. Koch, J. Olsen, and A.K. Wilson, Chem. Phys. Lett. 286, 243 (1998).

[34] T.H. Dunning, K.A. Peterson, and A.K. Wilson, J. Chem. Phys. 114, 9244 (2001).

[35] P. Botschwina, M. Horn, S. Seeger, and R. Oswald, Ber. Bunsenges. Phys. Chem. 101, 387 (1997). 
Table I: Harmonic and anharmonic frequencies $\left(\mathrm{cm}^{-1}\right)$ of formaldehyde obtained from multi-level potential energy surfaces (1D:CCSD(T)-2D:MP4(SDQ)-3D:MP2) spanned by normal coordinates obtained at different levels of electronic structure theory.

\begin{tabular}{|c|c|c|c|c|c|c|c|c|c|c|c|}
\hline \multirow[b]{2}{*}{$\nu_{i}$} & \multirow[b]{2}{*}{ Sym } & \multirow[b]{2}{*}{ Expt. ${ }^{c}$} & \multicolumn{3}{|c|}{$\mathrm{MP} 2^{a}$} & \multicolumn{3}{|c|}{$\mathrm{MP} 4(\mathrm{SDQ})^{a}$} & \multicolumn{3}{|c|}{$\operatorname{CCSD}(\mathrm{T})^{a}$} \\
\hline & & & Harm. & VSCF & VCI & Harm. & VSCF & VCI & Harm. & VSCF & VCI \\
\hline 1 & $\mathrm{a}_{1}$ & 2782.5 & 2970.0 & 2814.5 & 2780.5 & 2953.3 & 2814.1 & 2780.2 & 2929.0 & 2814.4 & 2780.1 \\
\hline 2 & & 1746.0 & 1771.5 & 1751.6 & 1748.5 & 1812.3 & 1751.9 & 1748.5 & 1779.9 & 1751.9 & 1748.6 \\
\hline 3 & & 1500.2 & 1553.2 & 1512.2 & 1506.6 & 1556.2 & 1511.3 & 1506.7 & 1542.8 & 1511.6 & 1506.8 \\
\hline 4 & $b_{2}$ & 2843.3 & 3043.0 & 2835.9 & 2836.6 & 3082.6 & 2835.4 & 2837.7 & 2995.6 & 2835.6 & 2838.3 \\
\hline 5 & & 1249.1 & 1280.1 & 1252.4 & 1248.0 & 1284.0 & 1252.4 & 1248.2 & 1274.6 & 1252.3 & 1248.2 \\
\hline 6 & $b_{1}$ & 1167.3 & 1208.6 & 1161.7 & 1159.9 & 1212.5 & 1160.3 & 1158.5 & 1192.0 & 1160.2 & 1158.4 \\
\hline \multicolumn{3}{|c|}{$\left|\Delta_{a v}\right|^{b}$} & 21.7 & 0.5 & 0.7 & 31.2 & 0.2 & 0.2 & 0.0 & 0.0 & 0.0 \\
\hline \multicolumn{3}{|c|}{$\left|\Delta_{\max }\right|^{b}$} & 47.4 & 1.5 & 1.7 & 87.0 & 0.3 & 0.6 & 0.0 & 0.0 & 0.0 \\
\hline
\end{tabular}

a) Level of electronic structure theory for calculating the normal coordinates.

b) Mean and maximum absolute deviation with respect to results obtained from calculations based on multi-level potential energy surfaces spanned by $\operatorname{CCSD}(\mathrm{T})$ normal coordinates (last three columns).

c) Experimental values were taken from Ref. [16]. 
Table II: Harmonic and anharmonic frequencies $\left(\mathrm{cm}^{-1}\right)$ of phosphorus trichloride obtained from multi-level potential energy surfaces (1D:CCSD(T)-2D:MP4(SDQ)-3D:MP2) spanned by normal coordinates obtained at different levels of electronic structure theory.

\begin{tabular}{|c|c|c|c|c|c|c|c|c|c|c|c|}
\hline \multirow[b]{2}{*}{$\nu_{i}$} & \multirow[b]{2}{*}{ Sym } & \multirow[b]{2}{*}{ Expt. $^{c}$} & \multicolumn{3}{|c|}{$\mathrm{MP} 2^{a}$} & \multicolumn{3}{|c|}{$\mathrm{MP} 4(\mathrm{SDQ})^{a}$} & \multicolumn{3}{|c|}{$\operatorname{CCSD}(\mathrm{T})^{a}$} \\
\hline & & & Harm. & $\mathrm{VSCF}$ & VCI & Harm. & VSCF & VCI & Harm. & VSCF & VCI \\
\hline 1 & $a_{1}$ & 504 & 515.7 & 513.6 & 511.8 & 522.7 & 513.5 & 511.6 & 528.3 & 513.4 & 511.3 \\
\hline 2 & & 252 & 256.3 & 255.2 & 254.9 & 261.0 & 255.2 & 254.9 & 261.1 & 255.2 & 254.9 \\
\hline 3 & e & 482 & 507.6 & 503.7 & 502.4 & 516.7 & 503.8 & 502.3 & 517.7 & 503.7 & 502.4 \\
\hline 4 & & 198 & 184.4 & 183.7 & 183.5 & 187.8 & 183.7 & 183.5 & 188.0 & 183.6 & 183.5 \\
\hline & $\left.a v\right|^{b}$ & & 7.8 & 0.1 & 0.1 & 1.7 & 0.1 & 0.1 & 0.0 & 0.0 & 0.0 \\
\hline & $\left.\max \right|^{b}$ & & 12.6 & 0.2 & 0.5 & 5.6 & 0.1 & 0.3 & 0.0 & 0.0 & 0.0 \\
\hline
\end{tabular}

a) Level of electronic structure theory for calculating the normal coordinates.

b) Mean and maximum absolute deviation with respect to results obtained from calculations based on multi-level potential energy surfaces spanned by $\operatorname{CCSD}(\mathrm{T})$ normal coordinates (last three columns).

c) Experimental values were taken from Ref. [17]. 
Table III: Harmonic and anharmonic frequencies $\left(\mathrm{cm}^{-1}\right)$ of dibromomethane obtained from multi-level potential energy surfaces (1D:CCSD(T)-2D:MP4(SDQ)-3D:MP2) spanned by normal coordinates obtained at different levels of electronic structure theory.

\begin{tabular}{|c|c|c|c|c|c|c|c|c|c|c|c|}
\hline \multirow{2}{*}{\multicolumn{2}{|c|}{$\nu_{i}$ Sym }} & \multirow[b]{2}{*}{ Expt. ${ }^{c}$} & \multicolumn{3}{|c|}{$\mathrm{MP}^{a}$} & \multicolumn{3}{|c|}{$\mathrm{MP} 4(\mathrm{SDQ})^{a}$} & \multicolumn{3}{|c|}{$\operatorname{CCSD}(\mathrm{T})^{a}$} \\
\hline & & & Harm. & $\mathrm{VSCF}$ & VCI & Harm. & $\mathrm{VSCF}$ & $\mathrm{VCI}$ & Harm. & VSCF & $\mathrm{VCI}$ \\
\hline 1 & $a_{1}$ & 3009 & 3161.1 & 3014.0 & 3000.5 & 3158.7 & 3014.0 & 2999.9 & 3138.3 & 3014.1 & 3001.2 \\
\hline 2 & & 1382 & 1455.7 & 1424.0 & 1409.9 & 1463.6 & 1423.8 & 1409.4 & 1446.5 & 1423.7 & 1409.7 \\
\hline 3 & & 588 & 606.5 & 583.4 & 580.2 & 598.1 & 583.7 & 580.4 & 589.0 & 583.8 & 580.5 \\
\hline 4 & & 169 & 174.4 & 169.2 & 169.8 & 173.4 & 169.2 & 169.8 & 170.8 & 169.1 & 169.8 \\
\hline 5 & $\mathrm{a}_{2}$ & 1095 & 1136.8 & 1115.4 & 1102.3 & 1136.7 & 1115.4 & 1102.1 & 1121.8 & 1115.4 & 1102.2 \\
\hline 6 & $b_{1}$ & 3073 & 3247.5 & 3061.0 & 3059.8 & 3241.6 & 3061.2 & 3059.8 & 3220.9 & 3061.2 & 3059.6 \\
\hline 7 & & 812 & 828.9 & 828.5 & 811.9 & 829.0 & 828.4 & 811.7 & 819.1 & 828.5 & 811.9 \\
\hline 8 & $\mathrm{~b}_{2}$ & 1195 & 1232.3 & 1213.7 & 1201.1 & 1241.0 & 1213.6 & 1200.9 & 1221.4 & 1213.6 & 1201.0 \\
\hline 9 & & 653 & 695.3 & 657.4 & 654.6 & 688.2 & 657.6 & 654.7 & 670.8 & 657.6 & 654.7 \\
\hline \multicolumn{3}{|c|}{$\left|\Delta_{a v}\right|^{b}$} & 15.5 & 0.2 & 0.2 & 14.6 & 0.1 & 0.3 & 0.0 & 0.0 & 0.0 \\
\hline \multicolumn{3}{|c|}{$\left|\Delta_{\max }\right|^{b}$} & 26.6 & 0.4 & 0.7 & 20.7 & 0.1 & 1.3 & 0.0 & 0.0 & 0.0 \\
\hline
\end{tabular}

a) Level of electronic structure theory for calculating the normal coordinates.

b) Mean and maximum absolute deviation with respect to results obtained from calculations based on multi-level potential energy surfaces spanned by $\operatorname{CCSD}(\mathrm{T})$ normal coordinates (last three columns).

c) Experimental values were taken from Refs. [18, 19]. 
Table IV: Harmonic and anharmonic frequencies $\left(\mathrm{cm}^{-1}\right)$ of vinyl chloride obtained from multi-level potential energy surfaces (1D:CCSD(T)-2D:MP4(SDQ)-3D:MP2) spanned by normal coordinates obtained at different levels of electronic structure theory.

\begin{tabular}{|c|c|c|c|c|c|c|c|c|c|c|}
\hline \multirow[b]{2}{*}{$\nu_{i} \mathrm{Sym}$} & \multirow[b]{2}{*}{ Expt. ${ }^{c}$} & \multicolumn{3}{|c|}{$\mathrm{MP} 2^{a}$} & \multicolumn{3}{|c|}{$\mathrm{MP} 4(\mathrm{SDQ})^{a}$} & \multicolumn{3}{|c|}{$\operatorname{CCSD}(\mathrm{T})^{a}$} \\
\hline & & Harm. & VSCF & VCI & Harm. & VSCF & VCI & Harm. & VSCF & VCI \\
\hline $1 \quad a^{\prime}$ & 3121 & 3306.3 & 3104.2 & 3102.3 & 3286.8 & 3105.5 & 3103.6 & 3261.6 & 3105.1 & 3105.3 \\
\hline 2 & 3087 & 3252.3 & 3049.9 & 3082.1 & 3247.0 & 3052.2 & 3080.8 & 3221.1 & 3051.8 & 3080.6 \\
\hline 3 & 3020 & 3200.0 & 3037.1 & 3017.1 & 3189.0 & 3037.8 & 3017.9 & 3163.3 & 3037.6 & 3019.0 \\
\hline 4 & 1610 & 1657.6 & 1618.3 & 1606.5 & 1684.3 & 1618.4 & 1608.0 & 1648.9 & 1618.6 & 1607.2 \\
\hline 5 & 1371 & 1414.8 & 1388.6 & 1365.3 & 1427.5 & 1388.5 & 1365.3 & 1408.6 & 1388.8 & 1366.6 \\
\hline 6 & 1280 & 1310.4 & 1284.3 & 1273.2 & 1320.7 & 1285.0 & 1273.6 & 1302.8 & 1284.8 & 1273.7 \\
\hline 7 & 1031 & 1047.4 & 1043.4 & 1022.4 & 1056.4 & 1043.5 & 1022.7 & 1042.4 & 1043.6 & 1022.9 \\
\hline 8 & 722 & 743.2 & 723.0 & 711.7 & 735.1 & 722.3 & 711.7 & 725.0 & 722.4 & 712.0 \\
\hline 9 & 398 & 396.7 & 405.0 & 390.8 & 397.5 & 404.6 & 391.3 & 391.5 & 404.7 & 391.6 \\
\hline a" & 942 & 987.4 & 971.4 & 943.2 & 991.2 & 971.2 & 943.4 & 966.8 & 971.5 & 943.3 \\
\hline 11 & 897 & 914.5 & 923.4 & 891.1 & 937.5 & 923.3 & 891.2 & 906.5 & 923.0 & 891.3 \\
\hline 12 & 620 & 640.4 & 655.7 & 617.6 & 638.4 & 655.8 & 618.0 & 624.1 & 655.4 & 617.7 \\
\hline$\left|\Delta_{a v}\right|^{b}$ & & 17.2 & 0.6 & 0.9 & 20.7 & 0.3 & 0.6 & 0.0 & 0.0 & 0.0 \\
\hline$\left|\Delta_{\max }\right|^{b}$ & & 44.7 & 1.9 & 3.0 & 35.4 & 0.4 & 1.7 & 0.0 & 0.0 & 0.0 \\
\hline
\end{tabular}

a) Level of electronic structure theory for calculating the normal coordinates.

b) Mean and maximum absolute deviation with respect to results obtained from calculations based on multi-level potential energy surfaces spanned by $\operatorname{CCSD}(\mathrm{T})$ normal coordinates (last three columns).

c) Experimental values were taken from Ref. [21]. 
Table V: Harmonic and anharmonic frequencies $\left(\mathrm{cm}^{-1}\right)$ of trans-difluoroethylene obtained from multi-level potential energy surfaces (1D:CCSD(T)-2D:MP4(SDQ)-3D:MP2) spanned by normal coordinates obtained at different levels of electronic structure theory.

\begin{tabular}{|c|c|c|c|c|c|c|c|c|c|c|}
\hline \multirow[b]{2}{*}{$\nu_{i}$ Sym } & \multirow[b]{2}{*}{ Expt. ${ }^{c}$} & \multicolumn{3}{|c|}{$\mathrm{MP}^{a}$} & \multicolumn{3}{|c|}{$\mathrm{MP} 4(\mathrm{SDQ})^{a}$} & \multicolumn{3}{|c|}{$\operatorname{CCSD}(\mathrm{T})^{a}$} \\
\hline & & Harm. & VSCF & VCI & Harm. & VSCF & VCI & Harm. & VSCF & VCI \\
\hline $1 \quad \mathrm{a}_{g}$ & 3109 & 3281.0 & 3115.9 & 3102.9 & 3272.4 & 3115.8 & 3102.9 & 3244.6 & 3115.9 & 3105.1 \\
\hline 2 & 1702 & 1762.0 & 1716.0 & 1706.2 & 1787.3 & 1716.2 & 1706.4 & 1749.3 & 1715.5 & 1706.2 \\
\hline 3 & 1286 & 1322.3 & 1295.6 & 1286.0 & 1330.8 & 1295.8 & 1286.3 & 1313.6 & 1295.5 & 1285.9 \\
\hline 4 & 1123 & 1173.7 & 1151.8 & 1146.5 & 1180.5 & 1151.8 & 1146.7 & 1167.5 & 1151.7 & 1146.4 \\
\hline 5 & 552 & 560.1 & 551.4 & 549.6 & 563.5 & 551.5 & 549.7 & 556.4 & 551.1 & 549.4 \\
\hline $\mathrm{a}_{u}$ & 874.2 & 925.7 & 911.1 & 888.0 & 931.3 & 911.3 & 887.8 & 907.5 & 910.9 & 887.5 \\
\hline 7 & 335 & 345.2 & 333.6 & 333.6 & 345.3 & 333.8 & 333.7 & 337.9 & 333.4 & 333.3 \\
\hline $\mathrm{b}_{g}$ & 788 & 824.0 & 806.6 & 785.1 & 834.3 & 806.8 & 785.4 & 799.9 & 806.4 & 785.3 \\
\hline 9 & 3101 & 3272.2 & 3086.9 & 3089.0 & 3262.6 & 3086.8 & 3089.7 & 3235.8 & 3086.4 & 3088.7 \\
\hline 10 & 1274.2 & 1314.4 & 1292.1 & 1279.5 & 1324.3 & 1292.6 & 1280.1 & 1310.1 & 1292.0 & 1279.6 \\
\hline 11 & 1159 & 1203.4 & 1176.8 & 1172.2 & 1208.1 & 1176.6 & 1172.0 & 1195.9 & 1176.6 & 1172.1 \\
\hline 12 & 324 & 316.9 & 320.4 & 315.3 & 320.7 & 320.6 & 315.5 & 314.6 & 320.2 & 315.2 \\
\hline$\left|\Delta_{a v}\right|^{b}$ & & 14.0 & 0.2 & 0.4 & 19.0 & 0.4 & 0.5 & 0.0 & 0.0 & 0.0 \\
\hline$\left|\Delta_{\max }\right|^{b}$ & & 36.4 & 0.5 & 2.2 & 34.4 & 0.7 & 2.2 & 0.0 & 0.0 & 0.0 \\
\hline
\end{tabular}

a) Level of electronic structure theory for calculating the normal coordinates.

b) Mean and maximum absolute deviation with respect to results obtained from calculations based on multi-level potential energy surfaces spanned by $\operatorname{CCSD}(\mathrm{T})$ normal coordinates (last three columns).

c) Experimental values were taken from Ref. [20].

URL: http://mc.manuscriptcentral.com/tandf/tmph 
Table VI: Anharmonic cs-VCI frequencies $\left(\mathrm{cm}^{-1}\right)$ of 1,2,5-oxadiazole obtained from multilevel potential energy surfaces (1D:CCSD(T)-2D:MP4(SDQ)-3D:MP2) spanned by normal coordinates obtained at different levels of electronic structure theory.

\begin{tabular}{|c|c|c|c|c|c|}
\hline \# Sym & $\mathrm{B} 3 \mathrm{LYP}^{a}$ & $\mathrm{MP} 2^{a}$ & $\mathrm{MP} 4(\mathrm{SDQ})^{a}$ & $\mathrm{QCISD}(\mathrm{T})^{a}$ & $\operatorname{CCSD}(\mathrm{T})^{a}$ \\
\hline$a_{1}$ & 3155.81 & 3151.81 & 3155.84 & 3151.74 & 3152.93 \\
\hline 2 & 1417.00 & 1413.23 & 1417.86 & 1417.66 & 1417.66 \\
\hline 3 & 1309.11 & 1319.59 & 1308.45 & 1309.55 & 1309.75 \\
\hline 4 & 1037.39 & 1039.42 & 1037.20 & 1038.52 & 1038.55 \\
\hline 5 & 1000.54 & 999.39 & 1000.52 & 999.68 & 999.77 \\
\hline 6 & 871.16 & 865.37 & 871.83 & 870.28 & 870.66 \\
\hline $\begin{array}{ll}7 & b_{2}\end{array}$ & 3132.79 & 3131.85 & 3134.16 & 3129.27 & 3129.57 \\
\hline 8 & 1530.20 & 1534.47 & 1529.54 & 1530.72 & 1530.89 \\
\hline 9 & 1174.94 & 1172.07 & 1173.13 & 1173.60 & 1173.56 \\
\hline 10 & 946.89 & 947.98 & 943.72 & 945.91 & 946.11 \\
\hline 11 & 832.91 & 831.64 & 841.62 & 835.98 & 835.70 \\
\hline $12 a_{2}$ & 890.16 & 891.74 & 890.65 & 890.26 & 890.55 \\
\hline 13 & 637.36 & 634.38 & 636.01 & 635.92 & 635.70 \\
\hline $14 b_{1}$ & 849.72 & 850.03 & 850.63 & 849.07 & 849.10 \\
\hline 15 & 628.80 & 626.80 & 628.87 & 628.65 & 628.63 \\
\hline$\left|\Delta_{a v}\right|^{a}$ & 1.2 & 2.7 & 1.6 & 0.2 & 0.0 \\
\hline$\left|\Delta_{\max }\right|^{b}$ & 3.2 & 9.8 & 5.9 & 1.2 & 0.0 \\
\hline
\end{tabular}

a) Level of electronic structure theory for calculating the normal coordinates.

b) Mean and maximum absolute deviation with respect to results obtained from cs-VCI calculations based on multi-level potential energy surfaces spanned by CCSD(T) normal coordinates (last column). 
Table VII: Optimized internal coordinates for CHFClI at various levels of theory. Distances $r_{e}$ in Ångstrom, bond and torsion angles, $\alpha_{e}$ and $\tau_{e}$, in degrees.

\begin{tabular}{|c|c|c|c|c|c|c|}
\hline \multirow[b]{2}{*}{ Parameter } & \multicolumn{2}{|c|}{ MP2 } & \multicolumn{4}{|c|}{$\operatorname{CCSD}(\mathrm{T})$} \\
\hline & avtz & avqz & avtz & avqz & $\mathrm{CBS}-3^{a}$ & $\mathrm{CBS}-2^{b}$ \\
\hline$r_{e}^{C H}$ & 1.0837 & 1.0832 & 1.0847 & 1.0843 & 1.0845 & 1.0840 \\
\hline$r_{e}^{C F}$ & 1.3549 & 1.3515 & 1.3550 & 1.3506 & 1.3482 & 1.3474 \\
\hline$r_{e}^{C C l}$ & 1.7574 & 1.7513 & 1.7671 & 1.7604 & 1.7563 & 1.7555 \\
\hline$r_{e}^{C I}$ & 2.1379 & 2.1398 & 2.1490 & 2.1513 & 2.1534 & 2.1529 \\
\hline$\alpha_{e}^{H C I}$ & 107.55 & 107.42 & 107.62 & 107.42 & & \\
\hline$\alpha_{e}^{F C I}$ & 109.50 & 109.42 & 109.46 & 109.39 & & \\
\hline$\alpha_{e}^{C l C I}$ & 112.18 & 112.21 & 112.27 & 112.29 & & \\
\hline$\tau_{e}^{C l C I H}$ & 119.45 & 119.49 & 119.43 & 119.42 & & \\
\hline$\tau_{e}^{F C I H}$ & -118.80 & -118.71 & -118.92 & -118.83 & & \\
\hline
\end{tabular}

a) Complete basis set limit using the three-parameter extrapolation formula (CBS-3) of Peterson et al. [31].

b) Complete basis set limit using the two-parameter formula (CBS-2) of Helgaker et al. $[32,33]$. 
Table VIII: Vibrationally averaged structures of CHFClI and CDFClI. Distances, $r_{z}$, in Angstrom, bond and torsion angles, $\alpha_{z}$ and $\tau_{z}$, in degrees.

\begin{tabular}{|c|c|c|c|c|}
\hline & \multicolumn{2}{|c|}{ CHFClI } & \multicolumn{2}{|c|}{ CDFClI } \\
\hline & $\mathrm{MP}^{a}$ & $\operatorname{CCSD}(\mathrm{T})^{a}$ & $\mathrm{MP} 2^{a}$ & $\operatorname{CCSD}(\mathrm{T})^{a}$ \\
\hline$r_{z}^{C H}$ & 1.0930 & 1.0930 & 1.0908 & 1.0908 \\
\hline$r_{z}^{C F}$ & 1.3603 & 1.3602 & 1.3602 & 1.3601 \\
\hline$r_{z}^{C C l}$ & 1.7732 & 1.7730 & 1.7727 & 1.7726 \\
\hline$r_{z}^{C I}$ & 2.1550 & 2.1550 & 2.1546 & 2.1545 \\
\hline$\alpha_{z}^{H C I}$ & 107.84 & 107.83 & 107.80 & 107.79 \\
\hline$\alpha_{z}^{F C I}$ & 109.32 & 109.32 & 109.28 & 109.36 \\
\hline$\alpha_{z}^{C l C I}$ & 112.16 & 112.16 & 112.20 & 112.20 \\
\hline$\tau_{z}^{C l C I H}$ & 119.67 & 119.66 & 119.63 & 119.61 \\
\hline$\tau_{z}^{F C I H}$ & -119.01 & -119.01 & -118.95 & -118.96 \\
\hline
\end{tabular}

a) Level of electronic structure theory for calculating the normal coordinates. The wavefunction of the vibrational ground state used for calculating the vibrationally averaged parameters was obtained from a multi-level potential (see text).

URL: http://mc.manuscriptcentral.com/tandf/tmph 22 
Table IX: Experimental and calculated rotational constants (in $\mathrm{MHz}$ ) for $\mathrm{CH}^{35} \mathrm{FClI}$ and $\mathrm{CH}^{37} \mathrm{FClI}$

\begin{tabular}{|c|c|c|c|c|c|c|c|}
\hline \multirow[b]{2}{*}{ Method } & \multirow[b]{2}{*}{ Type } & \multicolumn{3}{|c|}{$\mathrm{CH}^{35} \mathrm{FClI}$} & \multicolumn{3}{|c|}{$\mathrm{CH}^{37} \mathrm{FClI}$} \\
\hline & & $\mathrm{A}$ & B & $\mathrm{C}$ & A & $\mathrm{B}$ & $\mathrm{C}$ \\
\hline Exp. ${ }^{a}$ & Reference & 6278.65 & 1474.15 & 1224.41 & 6192.86 & 1432.70 & 1192.57 \\
\hline $\mathrm{CCSD}(\mathrm{T}) / \mathrm{avtz}$ & Equilibrium & 6248.61 & 1461.53 & 1215.06 & 6163.50 & 1420.27 & 1183.34 \\
\hline $\operatorname{CCSD}(\mathrm{T}) / \operatorname{avqz}$ & Equilibrium & 6282.95 & 1462.85 & 1217.11 & 6197.13 & 1421.64 & 1185.37 \\
\hline $\operatorname{CCSD}(\mathrm{T}) / \mathrm{CBS}^{-} 3^{b}$ & Equilibrium & 6304.97 & 1462.81 & 1217.84 & 6218.61 & 1421.66 & 1186.12 \\
\hline $\operatorname{CCSD}(\mathrm{T}) / \mathrm{CBS} 2^{c}$ & Equilibrium & 6311.32 & 1463.70 & 1218.66 & 6224.85 & 1422.53 & 1186.92 \\
\hline $\operatorname{CCSD}(\mathrm{T}) / \mathrm{avtz}$ & Vibr. averaged & 6207.64 & 1454.34 & 1209.12 & 6123.60 & 1413.23 & 1177.54 \\
\hline
\end{tabular}

a) Experimental value taken from Ref. [13].

b) Complete basis set limit using the three-parameter extrapolation formula (CBS-3) of Peterson et al. [31].

c) Complete basis set limit using the two-parameter formula (CBS-2) of Helgaker et al. [32,33]. 
1

2

3

4

5

6

7

8

9

10

11

12

13

14

15

16

17

18

19

20

21

22

23

24

25

26

27

28

29

30

31

32

33

34

35

36

37

38

39

40

41

42

43

44

45

46

47

48

49

50

51

52

53

54

55

56

57

58

59

60

Table X: Harmonic vibrational frequencies $\omega_{i}\left(\right.$ in $\mathrm{cm}^{-1}$ ) and intensities (in $\mathrm{km} / \mathrm{mol}$ ) for CHFCII.

\begin{tabular}{|c|c|c|c|c|c|}
\hline \multirow[b]{3}{*}{ Mode } & \multicolumn{4}{|c|}{ MP2 } & \multirow{3}{*}{$\begin{array}{r}\operatorname{CCSD}(\mathrm{T}) \\
\text { avtz } \\
\omega\end{array}$} \\
\hline & \multicolumn{2}{|c|}{ avtz } & \multicolumn{2}{|c|}{ avqz } & \\
\hline & $\omega$ & Int. & $\omega$ & Int. & \\
\hline$\omega_{1}$ & 3183.2 & 0.3 & 3182.7 & 0.3 & 3158.9 \\
\hline$\omega_{2}$ & 1332.0 & 9.0 & 1334.1 & 8.7 & 1326.7 \\
\hline$\omega_{3}$ & 1212.5 & 75.0 & 1212.6 & 73.7 & 1202.1 \\
\hline$\omega_{4}$ & 1083.1 & 172.5 & 1084.7 & 173.0 & 1087.6 \\
\hline$\omega_{5}$ & 797.6 & 163.9 & 802.8 & 162.6 & 782.2 \\
\hline$\omega_{6}$ & 615.5 & 32.5 & 610.3 & 35.0 & 602.2 \\
\hline$\omega_{7}$ & 419.6 & 0.4 & 421.5 & 0.4 & 414.0 \\
\hline$\omega_{8}$ & 278.0 & 0.3 & 277.3 & 0.3 & 273.7 \\
\hline$\omega_{9}$ & 197.1 & 0.0 & 197.3 & 0.0 & 193.1 \\
\hline
\end{tabular}

URL: http://mc.manuscriptcentral.com/tandf/tmph 24 
Table XI: Harmonic vibrational frequencies $\omega_{i}\left(\right.$ in $\left.\mathrm{cm}^{-1}\right)$ and intensities $($ in $\mathrm{km} / \mathrm{mol})$ for CDFClI.

\begin{tabular}{|c|c|c|c|c|c|}
\hline \multirow[b]{3}{*}{ Mode } & \multicolumn{4}{|c|}{ MP2 } & \multirow{3}{*}{$\begin{array}{r}\operatorname{CCSD}(\mathrm{T}) \\
\text { avtz } \\
\omega\end{array}$} \\
\hline & \multicolumn{2}{|c|}{ avtz } & \multicolumn{2}{|c|}{ avqz } & \\
\hline & $\omega$ & Int. & $\omega$ & Int. & \\
\hline$\omega_{1}$ & 2344.2 & 0.5 & 2343.8 & 0.5 & 2325.9 \\
\hline$\omega_{2}$ & 1086.5 & 170.8 & 1087.7 & 169.7 & 1091.8 \\
\hline$\omega_{3}$ & 985.0 & 8.8 & 987.7 & 9.0 & 980.8 \\
\hline$\omega_{4}$ & 921.2 & 147.0 & 920.3 & 147.9 & 912.9 \\
\hline$\omega_{5}$ & 762.3 & 89.8 & 766.7 & 87.7 & 747.8 \\
\hline$\omega_{6}$ & 572.4 & 21.1 & 568.2 & 22.9 & 559.9 \\
\hline$\omega_{7}$ & 417.5 & 0.4 & 419.4 & 0.5 & 412.0 \\
\hline$\omega_{8}$ & 277.5 & 0.3 & 276.8 & 0.3 & 273.2 \\
\hline$\omega_{9}$ & 196.4 & 0.0 & 196.6 & 0.0 & 192.4 \\
\hline
\end{tabular}

URL: http://mc.manuscriptcentral.com/tandf/tmph 25 
Table XII: Fundamental frequencies $\nu_{i}$ for CHFClI and CDFClI (in $\left.\mathrm{cm}^{-1}\right)$.

\begin{tabular}{lrrrrrr}
\hline \hline & \multicolumn{3}{c}{ CHFClI } & & \multicolumn{2}{c}{ CDFClI } \\
\cline { 2 - 3 } Mode & MP2 $^{a}$ & CCSD(T) $^{a}$ & Exp. $^{b}$ & & MP2 $^{a}$ & CCSD $(\mathrm{T})^{a}$ \\
\hline$\nu_{1}$ & 3014.9 & 3014.7 & 3010 & & 2255.4 & 2255.1 \\
$\nu_{2}$ & 1296.9 & 1297.4 & 1301 & & 1061.4 & 1061.8 \\
$\nu_{3}$ & 1179.4 & 1179.3 & 1180 & & 962.6 & 962.3 \\
$\nu_{4}$ & 1059.5 & 1059.6 & 1053 & & 895.9 & 895.7 \\
$\nu_{5}$ & 764.6 & 765.4 & 764 & & 731.9 & 733.0 \\
$\nu_{6}$ & 594.2 & 594.8 & 587 & & 554.2 & 554.2 \\
$\nu_{7}$ & 409.5 & 409.7 & 418 & & 407.6 & 407.6 \\
$\nu_{8}$ & 271.0 & 271.2 & 274 & & 270.5 & 270.6 \\
$\nu_{9}$ & 191.7 & 191.9 & 197 & & 191.1 & 191.1 \\
\hline \hline
\end{tabular}

a) Level of electronic structure theory for calculating the normal coordinates. The wavefunction of the vibrational ground state was obtained from a multi-level potential (see text).

b) Taken from Ref. 13. There is a misprint in Table 3 of Ref. 13. According to private communication with Dr. J. Crassous the given Raman frequency of $3150 \mathrm{~cm}^{-1}$ has to be replaced by $3010 \mathrm{~cm}^{-1}$. 


\title{
Vibrational Spectra Obtained from High Quality Potential Energy Surfaces Spanned by Low Level Normal Coordinates - Application to CHFClI and CDFCII
}

\author{
Sandra Heislbetz ${ }^{1}$, Peter Schwerdtfeger ${ }^{2}$, and Guntram Rauhut ${ }^{*, 1}$ \\ 1 Institut für Theoretische Chemie, Universität Stuttgart, Pfaffenwaldring 55, 70569 \\ Stuttgart, Germany. \\ ${ }^{2}$ Centre of Theoretical Chemistry and Physics (CTCP), Bldg. 44, Institute of Fundamen- \\ tal Sciences, Massey University (Auckland Campus), Private Bag 102904, North Shore \\ MSC, Auckland, New Zealand.
}

\section{Abstract}

The impact of approximate normal coordinates for spanning high level potential energy surfaces on vibrational frequencies is studied within the framework of vibrational SCF and configuration interaction calculations (VCI). The use of low level normal coordinates avoids expensive geometry optimizations and harmonic frequency calculations and thus allows for a significant reduction in CPU time. Benchmark calculations are provided for a set of molecules ranging from 5 to 7 atoms. An application to CHFClI and CDFClI shows that this approximation still allows for very accurate results. These molecules are of particular interest for studying parity violation effects.

*To whom correspondence should be addressed: rauhut@theochem.uni-stuttgart.de 


\section{Introduction}

The calculation of accurate vibrational spectra is a time consuming task. At reliable computational levels, e.g. CCSD (T)/aug-cc-p (C)VTZ and beyond, such calculations may require several weeks of CPU time even for small molecules and within the harmonic approximation. Consequently, most computational studies on vibrational spectra are based on density functional theory rather than wave function based $a b$ initio methods [1]. In most approaches for calculating anharmonic vibrational spectra, the computation of a reliable harmonic spectrum is a necessary prerequisite. Within perturbational approaches, the anharmonic corrections are simply added to the harmonic values [2-4], while for variational vibrational SCF calculations (VSCF) the normal modes of the harmonic calculation are often used within the expansion of the potential [5-7]. In perturbational calculations it appears to be common practice to compute the anharmonic corrections at a lower computational level than the harmonic frequencies $[1,3,4]$. In analogy to this, the expansion of the potential in terms of many mode representations can be performed at different computational levels as well, i.e. important one-mode terms (1D) need to be computed at the highest level, while for the less important three-mode or four-mode coupling terms (3D and 4D) lower theoretical levels appear to be sufficient $[8,9]$. The impact of the approximation was found to be very little once the different computational levels have been chosen carefully. For example, a 1D:CCSD(T)-2D:MP4(SDQ)-3D:MP2 multi-level scheme appears to be quite successful within the calculation of anharmonic vibrational spectra $[8,10]$. As the multidimensional potential calculated by a multi-level scheme does not refer exactly to the normal modes used for spanning the potential, the question arises if normal modes at lower computational levels can be used whithout introducing significant errors. Of course, this is not an approach for very accurate calculations (e.g. Ref. 11,12), but if the errors remain reasonably small the achieved accuracy may be sufficient for many applications while the overall CPU-time can be significantly reduced. Such a method would be appealing for two reasons: (1) low level calculations like MP2 or DFT are much faster than coupled-cluster calculations with a perturbational treatment of triple excitations. (2) For many low level methods analytical first and/or second order derivatives are available, while for the more sophisticated methods, e.g. density fitting $\operatorname{CCSD}(\mathrm{T})$, second derivatives need to be computed by twofold numerical differentiation which scales quadratically with respect to the degrees of freedom rather than linearly. 
Here we present a systematic study on the impact of different normal modes used for spanning multi-level potential energy surfaces on anharmonic vibrational frequencies. All calculations are based on configuration-selective vibrational CI calculations (cs-VCI) as will be described in detail below. In section 4 we present calculations for the fundamental modes of CHFClI and CDFClI which will be compared with the most recent experimental results of Soulard et al. [13]. These molecules are of particular interest as they are potential candidates for significant parity violation effects $[14,15]$. In addition, this study extends our work on chiral methane derivatives (see Ref. 10).

\section{Approximate normal coordinates}

\subsection{Computational details}

A small set of molecules ranging from 4 to 7 atoms has been used for studying the impact of the normal modes obtained from different electronic structure levels on the fundamental modes obtained from multi-level VCI calculations. This test set comprises formaldehyde [16], phosphorus trichloride [17], dibromomethane [18, 19], trans-difluoroethylene [20], vinyl chloride [21] and 1,2,5-oxadiazole [8,22]. Using a cc-pVTZ basis set throughout [23], all structures were optimized at the MP2, MP4(SDQ) and CCSD(T) levels of theory. The innermost 10 electrons of bromine (in dibromomethane) were approximately treated by a Stuttgart small core relativistic effective core potential ECP10MDF with a correlation consistent basis set adjusted by Peterson et al. [25]. Harmonic frequencies and thus the normal coordinates were computed at the same electronic structure levels. These coordinates were used for spanning multi-level 1D:CCSD(T)-2D:MP4(SDQ)-3D:MP2 potentials (see equation 1) which were applied in the subsequent variational VSCF and VCI calculations based on the Watson Hamiltonian [26]. 


$$
\begin{aligned}
V\left(q_{1}, \ldots, q_{3 N-6}\right)= & \underbrace{\sum_{i} V_{i}\left(q_{i}\right)}_{C C S D(T)}+\underbrace{\sum_{i<j} V_{i j}\left(q_{i}, q_{j}\right)}_{M P 4(S D Q)}+\underbrace{\sum_{i<j<k} V_{i j k}\left(q_{i}, q_{j}, q_{k}\right)}_{M P 2} \\
& +\underbrace{\sum_{i<j<k<l} V_{i j k l}\left(q_{i}, q_{j}, q_{k}, q_{l}\right)+\ldots}_{\text {neglected }}
\end{aligned}
$$

In all calculations presented here, this series has been truncated after the 3D terms, which was found to be sufficient for most applications. The correction due the $4 \mathrm{D}$ terms, which are fully implemented in our code, is very small. The difference potentials $V_{i}\left(q_{i}\right), V_{i j}\left(q_{i}, q_{j}\right)$ etc. are given as

$$
\begin{aligned}
V_{i}\left(q_{i}\right) & =V_{i}^{0}\left(q_{i}\right)-V_{0} \\
V_{i j}\left(q_{i}, q_{j}\right) & =V_{i j}^{0}\left(q_{i}, q_{j}\right)-\sum_{r \in\{i, j\}} V_{r}\left(q_{r}\right)-V_{0}
\end{aligned}
$$

and likewise for all other terms (3D and 4D). All quantities with the superscript 0 denote the total energy as obtained from the electronic structure calculations at distorted nuclear configurations. $V_{0}$ represents the total energy at the equilibrium geometry. In order to determine the incremental contributions in such a scheme correctly, one would in principle need to compute the one-mode (1D) contributions at all three levels and the $2 \mathrm{D}$ terms at the MP4(SDQ) and MP2 levels. This repeated calculation of the 1D and 2D grid points is necessary in order to generate consistent difference potentials which obey the conditions:

$$
V_{i j}\left(q_{i}, q_{j}=0\right)=V_{i j}\left(q_{i}=0, q_{j}\right)=0
$$

However, as in the MoLPRo suite of ab initio programs [27] the MP2 energy is a byproduct of the $\operatorname{CCSD}(\mathrm{T})$ and MP4(SDQ) computational schemes, in practice only the 1D terms need to be computed twice, i.e. at the $\operatorname{CCSD}(\mathrm{T})$ and MP4(SDQ) levels. Consequently, the additional computational effort is rather limited. The multi-level scheme has not been changed during this study. We explicitly note here, that this computational scheme usually is not sufficient to ensure an accuracy which results in excellent agreement with experimental data. Usually larger basis sets including diffuse functions [28], core correlation effects, and spin-orbit couplings appear to be important $[8,12]$. However, the computational scheme used here is accurate enough in order to exclude substantial 
artifacts and to allow for a comparison with experimental data. As reported in detail elsewhere [9] the potential energy surfaces of all molecules within this study were generated by a fully automated and parallelized algorithm which is based on an iterative interpolation technique and a prescreening scheme for the 3D contributions. Each difference potential is represented by 16 grid points in each dimension. These fine grids are obtained from one-dimensional polynomial interpolations based on an increasing number of coarse grid points. Within the VSCF approach single-mode vibrational wave functions (modals) were generated from 16 distributed Gaussians using a collocation algorithm [29]. Vibrational correlation effects were accounted for by a configuration-selective VCI approach, i.e. cs-VCI, including configurations with at most four different excited modals (quadruple excitations) while restricting the maximum excitation level within one modal to the fourth root. In addition, the overall excitation level was restricted to seven. From these initially generated configurations a subset has been selected for the final VCI calculations using a recently developed configuration selection scheme. All these algorithms are implemented in a development version of the MoLPRo suite of ab initio programs [27].

\subsection{Results}

Tables I - V show the results for the fundamental modes of the test set molecules except for 1,2,5-oxadiazole. In all these tables, the frequencies based on potentials spanned by CCSD(T)/cc-pVTZ normal coordinates were taken as the reference (last three columns). The harmonic MP2 and MP4(SDQ) frequencies usually lead to a strong systematic overestimation with respect to the $\operatorname{CCSD}(\mathrm{T})$ results. The mean absolute deviation for MP2 is $15.7 \mathrm{~cm}^{-1}$ with maximum deviations as large as $47.4 \mathrm{~cm}^{-1}$. Based on these results one is also tempted to expect rather significant deviations between the MP2 and CCSD(T) normal coordinates. However, calculation of the dot products of the normal coordinate displacement vectors obtained at different levels of electronic structure theory shows that this is not necessarily the case. As a result, the VSCF and cs-VCI frequencies obtained from potentials spanned by low level (i.e. MP2 or MP4(SDQ)) calculations usually closely resemble those obtained from $\operatorname{CCSD}(\mathrm{T})$ normal coordinates. For VSCF calculations, the mean absolute deviation is as low as $0.3 \mathrm{~cm}^{-1}$ for MP2 and $0.5 \mathrm{~cm}^{-1}$ for MP4(SDQ), respectively. For cs-VCI calculations this deviation is slightly larger: $0.5 \mathrm{~cm}^{-1}$ for MP2 
The substitution of $\operatorname{CCSD}(\mathrm{T})$ normal coordinates by low level coordinates is based on the assumption that both sets strongly resemble each other. The question arises, what happens if this cannot be guaranteed? For this very reason we have included 1,2,5oxadiazole into the test set. This is a molecule which is poorly represented by MP2 and even MP4(SDQ) calculations. For example, the CN bond lengths differ from the CCSD(T) bond length by $0.016 \AA$ at the MP2 level and by $0.010 \AA$ at the MP4(SDQ) level, respectively. Likewise, the CC bond length deviates at the MP2 level by $0.023 \AA$ from the coupled-cluster value. Dot products between the MP2 and the CCSD(T) normal coordinate displacement vectors can be as low as $0.81\left(6 a_{1}\right)$. Consequently, larger deviations than for the other test molecules must be anticipated in this case. VCI results for this molecule are provided in Table VI. As expected, a mean absolute deviation of $2.7 \mathrm{~cm}^{-1}$ at the MP2 level is far above the value for the other molecules and a maximum deviation of $9.8 \mathrm{~cm}^{-1}$ for the $3 a_{1}$ mode is not acceptable. This prompted us to use methods for calculating normal coordinates, which are not included in the multi-level approximation of the potential, but simply provide a reliable approximation to the coupled-cluster coordinates. Two methods appear to be particularly promising in that respect: (1) density functional methods which are known for a proper representation of vibrational frequencies and (2) the QCISD(T) method, which can be considered a truncated coupled-cluster variant [30]. QCISD $(\mathrm{T})$ calculations are not significantly faster than $\operatorname{CCSD}(\mathrm{T})$ calculations, but for the former one analytical gradients are available in MOLPRO which avoids the twofold numerical differentiation [24]. These results are also included in Table VI. Both 
methods yield results which are in excellent agreement with the coupled-cluster results. Furthermore, the deviations introduced by this approximation are significantly smaller than the error obtained from the limited basis set or the neglect of core-correlation effects. Consequently, it occurs that even problematic molecules can be handled within this approximation and thus expensive geometry optimizations and harmonic frequency calculations at the CCSD(T) appear not to be necessary for most systems.

The saving in CPU-time due to the introduced approximation can be quite substantial. As outlined above, the savings are due to 2 reasons: (1) the use of analytical first derivatives instead of a twofold numerical differentiation leads to a reduction from quadratic to linear scaling within the generation of the normal coordinates. (2) The formal scaling with respect to the molecular size of the low level correlation methods $\left(O\left(N^{5}\right)\right.$ for MP2 and $O\left(N^{4}\right)$ for DFT) is significantly better than for $\operatorname{CCSD}(\mathrm{T})$, i.e. $O\left(N^{7}\right)$. For the molecules of the test set this leads to speed-ups in the generation of the normal modes up to a factor of about 40. Consequently, the overall calculation time can be reduced significantly without introducing substantial errors.

\section{The vibrational spectra of CHFClI and CDFClI}

\subsection{Computational details}

The structure of CHFClI has been optimized at the MP2 and CCSD(T) levels in combination with aug-cc-pVTZ and aug-cc-pVQZ basis sets. Core correlation effects have not been included and the influence of the innermost 28 electrons of iodine was simulated by a Stuttgart small core relativistic effective core potential (ECP28MDF) with correlation consistent basis sets adjusted by Peterson [25]. Harmonic frequencies were computed for both isotopomers at the MP2/aug-cc-pVTZ, MP2/aug-cc-pVQZ and CCSD(T)/aug-ccpVTZ levels. While first analytical derivatives could be used for the MP2 calculation, the calculation of the $\operatorname{CCSD}(\mathrm{T})$ harmonic frequencies required a twofold numerical differentiation step. 


\subsection{Results}

Results for all internal coordinates of the equilibrium structure are provided in Table VII. The same trends are observed as in our previous study on CHFClBr [10]: In comparison to the MP2 results, CCSD(T) leads to slightly larger bond lengths. We address this general trend to the overestimation of correlation effects in MP2 calculations. Primarily this affects the $\mathrm{CCl}$ and $\mathrm{CI}$ bonds lengths. However, the overall agreement between the MP2 and CCSD(T) coordinates is excellent, in particular for the larger aug-cc-pVQZ basis set. Moreover, based on the $\operatorname{CCSD}(\mathrm{T}) /$ aug-cc-pVnZ, $n=2-4$, equilibrium bond lengths we have also computed the $r_{e}$ bond lengths for the complete basis set (CBS) limit using the three-parameter extrapolation formula of Peterson et al. [31] (denoted CBS-3) and the two-parameter equation (CBS-2) of Helgaker et al. [32,33]. These bond lengths must be considered the most accurate values. The two different approaches used for calculating the basis set limit provide an error bar of about $\pm 0.0005 \AA$. Surprisingly, the CBS-3 value for the $\mathrm{CH}$ bond is larger than the CCSD(T)/aug-cc-pVQZ result. This may originate from a rather short $\operatorname{CCSD}(\mathrm{T}) /$ aug-cc-pVTZ value which is already very close to the CCSD(T)/aug-cc-pVQZ bond length. However, the uncertainty of the CBS results is larger than the elongation with respect to the $\operatorname{CCSD}(\mathrm{T}) /$ aug-cc-pVQZ bond length. The CBS values indicate that except for the $\mathrm{CH}$ bond even the aug-cc-pVQZ results are far from being converged. The largest correction is found for the $\mathrm{CCl}$ bond length, but it is well known that for 2nd-row atoms the basis set exprapolation based on aug-cc-pVnZ 
bases may lead to nonnegligible errors [34] and thus we expect the CBS value for the $\mathrm{CCl}$ bond length to be less accurate than for the other bonds. In order to account for vibrational effects on the structural parameters, anharmonicity corrections were computed from the expectation value using the aug-cc-pVTZ multi-level VCI wavefunction of the vibrational ground-state. These corrections were applied to both, the MP2 and CCSD(T) equilibrium structures which refer to the aug-cc-pVTZ basis set. Results are shown in Table VIII. Here, the most important result is that the MP2 and $\operatorname{CCSD}(\mathrm{T})$ values results coincide due to the vibrational corrections - which of course is expected as long as the wave functions of the vibrational ground state are very similar. While these corrections are rather large in the MP2 case, they are significantly smaller for the CCSD(T) calculation. In other words, without performing a $\operatorname{CCSD}(\mathrm{T})$ geometry optimization, one is able to reproduce the coupled-cluster geometrical parameters - but of course in a more economic way. To the best of our knowledge experimental geometrical parameters are not available for this molecule. However, Soulard et al. [13] provide rotational constants for the $\mathrm{CH}^{35} \mathrm{ClFI}$ and $\mathrm{CH}^{37} \mathrm{FClI}$ isotopomers corresponding to vibrationally averaged geometries. Hence, we computed these constants for the equilibrium and the vibrationally averaged structures (cf. Table IX). The rotational constants were not computed via the expectation value of the eigenvalues of the moment of inertia tensor for the vibrational ground state but rather from the moment of inertia tensor of the vibrationally averaged structure. With respect to the equilibrium values still a considerable basis set dependence can be seen and for the A constant the CBS values still differ considerably from the augcc-pVQZ result. The two different methods for calculating the complete basis set limit again provide a measure for the uncertainty within these values. Empirical corrections to the $\mathrm{CCl}$ and $\mathrm{CI}$ bond lengths would yield slightly shorter bonds and thus larger rotational constants [35]. As our calculations do not include core-correlation effects, which - in line with the empirical corrections - usually lead to shorter bonds, we consider the CBS values provided in Table IX being slightly too low. However, as the experimental values and the computed values at the equilibrium geometry refer to different structures $\left(r_{e}\right.$ instead of $r_{z}$ ), this is not a fair comparison (although it is frequently done in the literature). Consequently, the only meaningful comparison is between the experimental values and the vibrationally averaged structure. The computed vibrationally averaged values underestimate the experimental values, indicating that the CCSD(T)/aug-cc-pVTZ structure is not yet fully converged. 
The harmonic frequencies and their corresponding intensities (obtained from analytical dipole moment derivatives) are listed in Tables X and XI. While basis sets (from aug-ccpVTZ to aug-cc-pVQZ) appear to be rather small (the maximum deviation $\Delta \omega_{\max }$ at the MP2 level is $5.2 \mathrm{~cm}^{-1}$ ), differences between MP2 and CCSD(T) are still quite substantial $\left(\Delta \omega_{\max }=24.3 \mathrm{~cm}^{-1}\right)$. While a direct comparison of the harmonic frequencies of CHFClI with the experimental results of Soulard et al. [13] (cf. Table XII) remains unsatisfying, it is interesting to note, that the lowest three vibrations $\left(\omega_{7}-\omega_{9}\right)$ are properly represented by both, the harmonic MP2 and the harmonic $\operatorname{CCSD}(\mathrm{T})$ calculations. Consequently, these modes appear to be less sensitive to electron correlation effects and are fairly harmonic in nature, while anharmonic corrections are important for all other modes. The overall agreement of the fundamental VCI modes (cf. Table XII) with the experimental values is excellent. The mean absolute deviation is $4.6 \mathrm{~cm}^{-1}$ while the maximum deviation is about twice as large $\left(8.3 \mathrm{~cm}^{-1}\right)$. The approximation introduced above for reducing the computational effort within the calculation of the normal modes essentially leads to negligible deviations for both isotopomers (mean absolute deviation: $0.3 \mathrm{~cm}^{-1}$, maximum deviation (CCl stretching mode): $1.1 \mathrm{~cm}^{-1}$ ). This result is not unexpected as the MP2 structural parameters closely resemble the $\operatorname{CCSD}(\mathrm{T})$ results (see above). On the other hand, the savings in CPU-time amount to about 20 days for this system, when running the harmonic frequency calculations on just one processor. No experimental values are available for CDFCII in the literature. Therefore, the data compiled here must be considered a prediction. However, we do not anticipate any larger errors for this isotopomer than for CHFClI. On the contrary, in analogy to our previous study on CHFClBr and CDFClBr we expect slightly smaller deviations with respect to experimentally observed values.

\section{Summary and Conclusions}

It could be shown that for the calculation of accurate vibrational spectra, it is not necessary to optimize the structural parameters of molecules at the highest levels of electronic structure theory. As long as the structural parameters of a molecule obtained by a low level 
method closely resemble those of high level methods, e.g. CCSD(T)/aug-cc-pVTZ (typically deviations should be less than 1\%), it appears that despite considerable differences in the harmonic frequencies the impact of the low level normal coordinates on the VCI frequencies is rather small - a very few wavenumbers only. Modern density functionals, which are known to be particular successful in the reproduction of structural parameters and harmonic vibrational spectra, appear to be the most promising low-level approaches for the generation of approximate normal coordinates. Even for small molecules, this may result in significant time savings. For the systems presented here, the fairly accurate multi-level VCI calculations (i.e. surface generation plus a vibrational CI calculation) usually takes about as long as a corresponding high-level harmonic frequency calculation. However, it must be admitted that this ratio holds only true as long as a twofold numerical differentiation at the high level cannot be avoided.

The structure and vibrational frequencies of CHFCII and CDFCII have been studied by accurate calculations up to the CCSD(T)/aug-cc-pVQZ level. Fundamental frequencies obtained from multi-level vibrational CI calculations are in excellent agreement with recent experimental results of Soulard et al. [13] for CHFClI. Maximum deviations are as low as $8.3 \mathrm{~cm}^{-1}$. In addition to that we present here predictions for the deuterated isotopomer.

\section{Acknowledgements}

This work was performed within the Collaborative Research Centre SFB 706 (Selective Catalytic Oxidations Using Molecular Oxygen; Stuttgart) and funded by the German Research Foundation. The authors thank Prof. P. Botschwina for helpful discussions and Dr. J. Crassous for a copy of the Raman spectrum of CHFClI. P. Schwerdtfeger acknowledges financial support from Marsden fund and the ISAT Linkage fund (Wellington). 


\section{References}

[1] A.D. Boese and J.M.L. Martin, J. Phys. Chem. A 108, 3085 (2004).

[2] D.A. Clabo Jr., W.D. Allen, R.B. Remington, Y. Yamaguchi, and H.F. Schaefer, Chem. Phys. 123, 187 (1988).

[3] H. Bürger, R. Kuna, S. Ma, J. Breidung, and W. Thiel, J. Chem. Phys. 101, 1 (1994).

[4] H. Bürger, S. Ma, J. Breidung, and W. Thiel, J. Chem. Phys. 104, 4945 (1996).

[5] J.M. Bowman, Acc. Chem. Res. 19, 202 (1986).

[6] J.M. Bowman, J. Chem. Phys. 68, 608 (1978).

[7] R. Gerber and M. Ratner, Adv. Chem. Phys. 70, 97 (1988).

[8] K. Pflüger, M. Paulus, S. Jagiella, T. Burkert, and G. Rauhut, Theor. Chem. Acc. 114, 327 (2005).

[9] G. Rauhut, J. Chem. Phys. 121, 9313 (2004).

[10] G. Rauhut, V. Barone, and P. Schwerdtfeger, J. Chem. Phys. 125, 054308 (2006).

[11] S.N. Yurchenko, J. Breidung and W. Thiel, Theor. Chem. Acc. 114, 333 (2005).

[12] J. Koput and K.A. Peterson, J. Chem. Phys. 125, 044306 (2006). 
[13] P. Soulard, P. Asselin, A. Cuisset, J.R.A. Moreno, T.R. Huet, D. Petitprez, J. Demaison, T.B. Freedman, X. Cao, L.A. Nafie, and J. Crassous, Phys. Chem. Chem. Phys. 8, 79 (2006).

[14] P. Schwerdtfeger, J.K. Laerdahl, and C. Chardonnet, Phys. Rev. A 65, 042508 (2002).

[15] P. Schwerdtfeger, T. Saue, J.N.P. van Stralen, and L. Visscher, Phys. Rev. A 71, 012103 (2005).

[16] D.E. Reisner, R.W. Field, J.L. Kinsey, and H.-L. Dai, J. Chem. Phys. 80, 5968 (1984).

[17] A. Ruoff, H. Burger, S. Biedermann, and J. Cichon, Spectrochim. Acta Part A 28, 953 (1972).

[18] R.S. Dennen, E.A. Piotrowski, and F.F. Cleveland, J. Chem. Phys. 49, 4385 (1968).

[19] C. Lugez, A. Shriver, L. Shriver-Mazzuoli, E. Lasson, and C.J. Nielsen, J. Chem. Phys. 97, 11617 (1993).

[20] N.C. Craig, K.L. Petersen, and D.C. McKean, J. Phys. Chem. A 106, 6358 (2002).

[21] W.A. Herrebout and B.J. van der Veken, J. Chem. Soc., Faraday Trans. 93, 3453 (1997).

[22] D.H. Christensen and O.F. Nielsen, J. Mol. Spectrosc. 24, 477 (1967).

[23] T.H. Dunning, J. Chem. Phys. 90, 1007 (1989).

[24] G. Rauhut and H.J. Werner, Phys. Chem. Chem. Phys. 3, 4853 (2001).

[25] K.A. Peterson, D. Figgen, E. Goll, H. Stoll, and M. Dolg, J. Chem. Phys. 119, 11113 (2003). 
[26] J.K.G. Watson, Mol. Phys. 15, 479 (1968).

[27] Molpro, Development Version 2006.2, is a package of ab initio programs written by H.-J. Werner and P. J. Knowles, with contributions from R. D. Amos, A. Bernhardsson, A. Berning, P. Celani, D. L. Cooper, M. J. O. Deegan, A. J. Dobbyn, F. Eckert, C. Hampel, G. Hetzer, T. Korona, R. Lindh, A. W. Lloyd, F. R. Manby, W. Meyer, S. J. McNicholas, M. E. Mura, A. Nicklass, P. Palmieri, K. Peterson, P. Pulay, G. Rauhut, M. Schütz, U. Schumann, H. Stoll, A. J. Stone, R. Tarroni, and T. Thorsteinsson., (see http://www.molpro.net/).

[28] R.A. Kendall, T.H. Dunning, and R.J. Harrison, J. Chem. Phys. 96, 6769 (1992).

[29] W. Yang and A.C. Peet, Chem. Phys. Lett. 153, 98 (1988).

[30] J.A. Pople, M. Head-Gordon, and K. Raghavachari, J. Chem. Phys. 87, 5968 (1987).

[31] K.A. Peterson, D.E. Woon, and T.H. Dunning, J. Chem. Phys. 100, 7410 (1994).

[32] T. Helgaker, W. Klopper, H. Koch, and J. Noga, J. Chem. Phys. 106, 9639 (1997).

[33] A. Halkier, T. Helgaker, P. Jørgensen, W. Klopper, H. Koch, J. Olsen, and A.K. Wilson,

Chem. Phys. Lett. 286, 243 (1998).

[34] T.H. Dunning, K.A. Peterson, and A.K. Wilson, J. Chem. Phys. 114, 9244 (2001).

[35] P. Botschwina, M. Horn, S. Seeger, and R. Oswald, Ber. Bunsenges. Phys. Chem. 101, 387 (1997). 
Table I: Harmonic and anharmonic frequencies $\left(\mathrm{cm}^{-1}\right)$ of formaldehyde obtained from multi-level potential energy surfaces (1D:CCSD(T)-2D:MP4(SDQ)-3D:MP2) spanned by normal coordinates obtained at different levels of electronic structure theory.

\begin{tabular}{|c|c|c|c|c|c|c|c|c|c|c|c|}
\hline \multirow[b]{2}{*}{$\nu_{i}$} & \multirow[b]{2}{*}{ Sym } & \multirow[b]{2}{*}{ Expt. $^{c}$} & \multicolumn{3}{|c|}{$\mathrm{MP}^{a}$} & \multicolumn{3}{|c|}{$\mathrm{MP} 4(\mathrm{SDQ})^{a}$} & \multicolumn{3}{|c|}{$\operatorname{CCSD}(\mathrm{T})^{a}$} \\
\hline & & & Harm. & VSCF & VCI & Harm. & VSCF & VCI & Harm. & VSCF & $\mathrm{VCI}$ \\
\hline 1 & $a_{1}$ & 2782.5 & 2970.0 & 2814.5 & 2780.5 & 2953.3 & 2814.1 & 2780.2 & 2929.0 & 2814.4 & 2780.1 \\
\hline 2 & & 1746.0 & 1771.5 & 1751.6 & 1748.5 & 1812.3 & 1751.9 & 1748.5 & 1779.9 & 1751.9 & 1748.6 \\
\hline 3 & & 1500.2 & 1553.2 & 1512.2 & 1506.6 & 1556.2 & 1511.3 & 1506.7 & 1542.8 & 1511.6 & 1506.8 \\
\hline 4 & $\mathrm{~b}_{2}$ & 2843.3 & 3043.0 & 2835.9 & 2836.6 & 3082.6 & 2835.4 & 2837.7 & 2995.6 & 2835.6 & 2838.3 \\
\hline 5 & & 1249.1 & 1280.1 & 1252.4 & 1248.0 & 1284.0 & 1252.4 & 1248.2 & 1274.6 & 1252.3 & 1248.2 \\
\hline 6 & $\mathrm{~b}_{1}$ & 1167.3 & 1208.6 & 1161.7 & 1159.9 & 1212.5 & 1160.3 & 1158.5 & 1192.0 & 1160.2 & 1158.4 \\
\hline \multicolumn{3}{|c|}{$\left|\Delta_{a v}\right|^{b}$} & 21.7 & 0.5 & 0.7 & 31.2 & 0.2 & 0.2 & 0.0 & 0.0 & 0.0 \\
\hline \multicolumn{3}{|c|}{$\left|\Delta_{\max }\right|^{b}$} & 47.4 & 1.5 & 1.7 & 87.0 & 0.3 & 0.6 & 0.0 & 0.0 & 0.0 \\
\hline
\end{tabular}

a) Level of electronic structure theory for calculating the normal coordinates.

b) Mean and maximum absolute deviation with respect to results obtained from calculations based on multi-level potential energy surfaces spanned by $\operatorname{CCSD}(\mathrm{T})$ normal coordinates (last three columns).

c) Experimental values were taken from Ref. [16]. 
Table II: Harmonic and anharmonic frequencies $\left(\mathrm{cm}^{-1}\right)$ of phosphorus trichloride obtained from multi-level potential energy surfaces (1D:CCSD(T)-2D:MP4(SDQ)-3D:MP2) spanned by normal coordinates obtained at different levels of electronic structure theory.

\begin{tabular}{|c|c|c|c|c|c|c|c|c|c|c|c|}
\hline \multirow[b]{2}{*}{$\nu_{i}$} & \multirow[b]{2}{*}{ Sym } & \multirow[b]{2}{*}{ Expt. $^{c}$} & \multicolumn{3}{|c|}{$\mathrm{MP}^{a}$} & \multicolumn{3}{|c|}{$\mathrm{MP}(\mathrm{SDQ})^{a}$} & \multicolumn{3}{|c|}{$\operatorname{CCSD}(\mathrm{T})^{a}$} \\
\hline & & & Harm. & VSCF & VCI & Harm. & VSCF & VCI & Harm. & VSCF & VCI \\
\hline 1 & $a_{1}$ & 504 & 515.7 & 513.6 & 511.8 & 522.7 & 513.5 & 511.6 & 528.3 & 513.4 & 511.3 \\
\hline 2 & & 252 & 256.3 & 255.2 & 254.9 & 261.0 & 255.2 & 254.9 & 261.1 & 255.2 & 254.9 \\
\hline 3 & e & 482 & 507.6 & 503.7 & 502.4 & 516.7 & 503.8 & 502.3 & 517.7 & 503.7 & 502.4 \\
\hline 4 & & 198 & 184.4 & 183.7 & 183.5 & 187.8 & 183.7 & 183.5 & 188.0 & 183.6 & 183.5 \\
\hline \multicolumn{3}{|c|}{$\left|\Delta_{a v}\right|^{b}$} & 7.8 & 0.1 & 0.1 & 1.7 & 0.1 & 0.1 & 0.0 & 0.0 & 0.0 \\
\hline \multicolumn{3}{|c|}{$\left|\Delta_{\max }\right|^{b}$} & 12.6 & 0.2 & 0.5 & 5.6 & 0.1 & 0.3 & 0.0 & 0.0 & 0.0 \\
\hline
\end{tabular}

a) Level of electronic structure theory for calculating the normal coordinates.

b) Mean and maximum absolute deviation with respect to results obtained from calculations based on multi-level potential energy surfaces spanned by $\operatorname{CCSD}(\mathrm{T})$ normal coordinates (last three columns).

c) Experimental values were taken from Ref. [17]. 
Table III: Harmonic and anharmonic frequencies $\left(\mathrm{cm}^{-1}\right)$ of dibromomethane obtained from multi-level potential energy surfaces (1D:CCSD(T)-2D:MP4(SDQ)-3D:MP2) spanned by normal coordinates obtained at different levels of electronic structure theory.

\begin{tabular}{|c|c|c|c|c|c|c|c|c|c|c|c|}
\hline \multirow[b]{2}{*}{$\nu_{i}$} & \multirow{2}{*}{ Sym } & \multirow[b]{2}{*}{ Expt. ${ }^{c}$} & \multicolumn{3}{|c|}{$\mathrm{MP}^{a}$} & \multicolumn{3}{|c|}{$\mathrm{MP} 4(\mathrm{SDQ})^{a}$} & \multicolumn{3}{|c|}{$\operatorname{CCSD}(\mathrm{T})^{a}$} \\
\hline & & & Harm. & VSCF & VCI & Harm. & VSCF & VCI & Harm. & $\mathrm{VSCF}$ & VCI \\
\hline 1 & $a_{1}$ & 3009 & 3161.1 & 3014.0 & 3000.5 & 3158.7 & 3014.0 & 2999.9 & 3138.3 & 3014.1 & 3001.2 \\
\hline 2 & & 1382 & 1455.7 & 1424.0 & 1409.9 & 1463.6 & 1423.8 & 1409.4 & 1446.5 & 1423.7 & 1409.7 \\
\hline 3 & & 588 & 606.5 & 583.4 & 580.2 & 598.1 & 583.7 & 580.4 & 589.0 & 583.8 & 580.5 \\
\hline 4 & & 169 & 174.4 & 169.2 & 169.8 & 173.4 & 169.2 & 169.8 & 170.8 & 169.1 & 169.8 \\
\hline 5 & $a_{2}$ & 1095 & 1136.8 & 1115.4 & 1102.3 & 1136.7 & 1115.4 & 1102.1 & 1121.8 & 1115.4 & 1102.2 \\
\hline 6 & $b_{1}$ & 3073 & 3247.5 & 3061.0 & 3059.8 & 3241.6 & 3061.2 & 3059.8 & 3220.9 & 3061.2 & 3059.6 \\
\hline 7 & & 812 & 828.9 & 828.5 & 811.9 & 829.0 & 828.4 & 811.7 & 819.1 & 828.5 & 811.9 \\
\hline 8 & $b_{2}$ & 1195 & 1232.3 & 1213.7 & 1201.1 & 1241.0 & 1213.6 & 1200.9 & 1221.4 & 1213.6 & 1201.0 \\
\hline 9 & & 653 & 695.3 & 657.4 & 654.6 & 688.2 & 657.6 & 654.7 & 670.8 & 657.6 & 654.7 \\
\hline \multicolumn{3}{|c|}{$\left|\Delta_{a v}\right|^{b}$} & 15.5 & 0.2 & 0.2 & 14.6 & 0.1 & 0.3 & 0.0 & 0.0 & 0.0 \\
\hline \multicolumn{3}{|c|}{$\left|\Delta_{\max }\right|^{b}$} & 26.6 & 0.4 & 0.7 & 20.7 & 0.1 & 1.3 & 0.0 & 0.0 & 0.0 \\
\hline
\end{tabular}

a) Level of electronic structure theory for calculating the normal coordinates.

b) Mean and maximum absolute deviation with respect to results obtained from calculations based on multi-level potential energy surfaces spanned by $\operatorname{CCSD}(\mathrm{T})$ normal coordinates (last three columns).

c) Experimental values were taken from Refs. $[18,19]$. 
Table IV: Harmonic and anharmonic frequencies $\left(\mathrm{cm}^{-1}\right)$ of vinyl chloride obtained from multi-level potential energy surfaces (1D:CCSD(T)-2D:MP4(SDQ)-3D:MP2) spanned by normal coordinates obtained at different levels of electronic structure theory.

\begin{tabular}{|c|c|c|c|c|c|c|c|c|c|c|}
\hline \multirow[b]{2}{*}{$\nu_{i}$} & \multirow[b]{2}{*}{ Expt. $^{c}$} & \multicolumn{3}{|c|}{$\mathrm{MP} 2^{a}$} & \multicolumn{3}{|c|}{$\mathrm{MP} 4(\mathrm{SDQ})^{a}$} & \multicolumn{3}{|c|}{$\operatorname{CCSD}(\mathrm{T})^{a}$} \\
\hline & & Harm. & VSCF & VCI & Harm. & $\mathrm{VSCF}$ & VCI & Harm. & VSCF & VCI \\
\hline 1 & 3121 & 3306.3 & 3104.2 & 3102.3 & 3286.8 & 3105.5 & 3103.6 & 3261.6 & 3105.1 & 3105.3 \\
\hline 2 & 3087 & 3252.3 & 3049.9 & 3082.1 & 3247.0 & 3052.2 & 3080.8 & 3221.1 & 3051.8 & 3080.6 \\
\hline 3 & 3020 & 3200.0 & 3037.1 & 3017.1 & 3189.0 & 3037.8 & 3017.9 & 3163.3 & 3037.6 & 3019.0 \\
\hline 4 & 1610 & 1657.6 & 1618.3 & 1606.5 & 1684.3 & 1618.4 & 1608.0 & 1648.9 & 1618.6 & 1607.2 \\
\hline 5 & 1371 & 1414.8 & 1388.6 & 1365.3 & 1427.5 & 1388.5 & 1365.3 & 1408.6 & 1388.8 & 1366.6 \\
\hline 6 & 1280 & 1310.4 & 1284.3 & 1273.2 & 1320.7 & 1285.0 & 1273.6 & 1302.8 & 1284.8 & 1273.7 \\
\hline 7 & 1031 & 1047.4 & 1043.4 & 1022.4 & 1056.4 & 1043.5 & 1022.7 & 1042.4 & 1043.6 & 1022.9 \\
\hline 8 & 722 & 743.2 & 723.0 & 711.7 & 735.1 & 722.3 & 711.7 & 725.0 & 722.4 & 712.0 \\
\hline 9 & 398 & 396.7 & 405.0 & 390.8 & 397.5 & 404.6 & 391.3 & 391.5 & 404.7 & 391.6 \\
\hline a" & 942 & 987.4 & 971.4 & 943.2 & 991.2 & 971.2 & 943.4 & 966.8 & 971.5 & 943.3 \\
\hline 11 & 897 & 914.5 & 923.4 & 891.1 & 937.5 & 923.3 & 891.2 & 906.5 & 923.0 & 891.3 \\
\hline 12 & 620 & 640.4 & 655.7 & 617.6 & 638.4 & 655.8 & 618.0 & 624.1 & 655.4 & 617.7 \\
\hline$\left|\Delta_{a v}\right|^{b}$ & & 17.2 & 0.6 & 0.9 & 20.7 & 0.3 & 0.6 & 0.0 & 0.0 & 0.0 \\
\hline$\left|\Delta_{\max }\right|^{b}$ & & 44.7 & 1.9 & 3.0 & 35.4 & 0.4 & 1.7 & 0.0 & 0.0 & 0.0 \\
\hline
\end{tabular}

a) Level of electronic structure theory for calculating the normal coordinates.

b) Mean and maximum absolute deviation with respect to results obtained from calculations based on multi-level potential energy surfaces spanned by CCSD(T) normal coordinates (last three columns).

c) Experimental values were taken from Ref. [21]. 
Table V: Harmonic and anharmonic frequencies $\left(\mathrm{cm}^{-1}\right)$ of trans-difluoroethylene obtained from multi-level potential energy surfaces (1D:CCSD(T)-2D:MP4(SDQ)-3D:MP2) spanned by normal coordinates obtained at different levels of electronic structure theory.

\begin{tabular}{|c|c|c|c|c|c|c|c|c|c|c|c|}
\hline \multirow[b]{2}{*}{$\nu_{i}$} & \multirow[b]{2}{*}{ Sym } & \multirow[b]{2}{*}{ Expt. ${ }^{c}$} & \multicolumn{3}{|c|}{$\mathrm{MP}^{a}$} & \multicolumn{3}{|c|}{$\mathrm{MP} 4(\mathrm{SDQ})^{a}$} & \multicolumn{3}{|c|}{$\operatorname{CCSD}(\mathrm{T})^{a}$} \\
\hline & & & Harm. & VSCF & VCI & Harm. & VSCF & VCI & Harm. & VSCF & VCI \\
\hline 1 & $\mathrm{a}_{g}$ & 3109 & 3281.0 & 3115.9 & 3102.9 & 3272.4 & 3115.8 & 3102.9 & 3244.6 & 3115.9 & 3105.1 \\
\hline 2 & & 1702 & 1762.0 & 1716.0 & 1706.2 & 1787.3 & 1716.2 & 1706.4 & 1749.3 & 1715.5 & 1706.2 \\
\hline 3 & & 1286 & 1322.3 & 1295.6 & 1286.0 & 1330.8 & 1295.8 & 1286.3 & 1313.6 & 1295.5 & 1285.9 \\
\hline 4 & & 1123 & 1173.7 & 1151.8 & 1146.5 & 1180.5 & 1151.8 & 1146.7 & 1167.5 & 1151.7 & 1146.4 \\
\hline 5 & & 552 & 560.1 & 551.4 & 549.6 & 563.5 & 551.5 & 549.7 & 556.4 & 551.1 & 549.4 \\
\hline 6 & $\mathrm{a}_{u}$ & 874.2 & 925.7 & 911.1 & 888.0 & 931.3 & 911.3 & 887.8 & 907.5 & 910.9 & 887.5 \\
\hline 7 & & 335 & 345.2 & 333.6 & 333.6 & 345.3 & 333.8 & 333.7 & 337.9 & 333.4 & 333.3 \\
\hline 8 & $\mathrm{~b}_{g}$ & 788 & 824.0 & 806.6 & 785.1 & 834.3 & 806.8 & 785.4 & 799.9 & 806.4 & 785.3 \\
\hline 9 & & 3101 & 3272.2 & 3086.9 & 3089.0 & 3262.6 & 3086.8 & 3089.7 & 3235.8 & 3086.4 & 3088.7 \\
\hline 10 & & 1274.2 & 1314.4 & 1292.1 & 1279.5 & 1324.3 & 1292.6 & 1280.1 & 1310.1 & 1292.0 & 1279.6 \\
\hline 11 & & 1159 & 1203.4 & 1176.8 & 1172.2 & 1208.1 & 1176.6 & 1172.0 & 1195.9 & 1176.6 & 1172.1 \\
\hline 12 & & 324 & 316.9 & 320.4 & 315.3 & 320.7 & 320.6 & 315.5 & 314.6 & 320.2 & 315.2 \\
\hline \multicolumn{3}{|c|}{$\left|\Delta_{a v}\right|^{b}$} & 14.0 & 0.2 & 0.4 & 19.0 & 0.4 & 0.5 & 0.0 & 0.0 & 0.0 \\
\hline \multicolumn{3}{|c|}{$\left|\Delta_{\max }\right|^{b}$} & 36.4 & 0.5 & 2.2 & 34.4 & 0.7 & 2.2 & 0.0 & 0.0 & 0.0 \\
\hline
\end{tabular}

a) Level of electronic structure theory for calculating the normal coordinates.

b) Mean and maximum absolute deviation with respect to results obtained from calculations based on multi-level potential energy surfaces spanned by CCSD(T) normal coordinates (last three columns).

c) Experimental values were taken from Ref. [20]. 
Table VI: Anharmonic cs-VCI frequencies $\left(\mathrm{cm}^{-1}\right)$ of 1,2,5-oxadiazole obtained from multilevel potential energy surfaces (1D:CCSD(T)-2D:MP4(SDQ)-3D:MP2) spanned by normal coordinates obtained at different levels of electronic structure theory.

\begin{tabular}{|c|c|c|c|c|c|}
\hline \# Sym & $\mathrm{B} 3 \mathrm{LYP}^{a}$ & $\mathrm{MP}^{a}$ & $\mathrm{MP} 4(\mathrm{SDQ})^{a}$ & $\mathrm{QCISD}(\mathrm{T})^{a}$ & $\operatorname{CCSD}(\mathrm{T})^{a}$ \\
\hline $1 a_{1}$ & 3155.81 & 3151.81 & 3155.84 & 3151.74 & 3152.93 \\
\hline 2 & 1417.00 & 1413.23 & 1417.86 & 1417.66 & 1417.66 \\
\hline 3 & 1309.11 & 1319.59 & 1308.45 & 1309.55 & 1309.75 \\
\hline 4 & 1037.39 & 1039.42 & 1037.20 & 1038.52 & 1038.55 \\
\hline 5 & 1000.54 & 999.39 & 1000.52 & 999.68 & 999.77 \\
\hline 6 & 871.16 & 865.37 & 871.83 & 870.28 & 870.66 \\
\hline $7 \quad b_{2}$ & 3132.79 & 3131.85 & 3134.16 & 3129.27 & 3129.57 \\
\hline 8 & 1530.20 & 1534.47 & 1529.54 & 1530.72 & 1530.89 \\
\hline 9 & 1174.94 & 1172.07 & 1173.13 & 1173.60 & 1173.56 \\
\hline 10 & 946.89 & 947.98 & 943.72 & 945.91 & 946.11 \\
\hline 11 & 832.91 & 831.64 & 841.62 & 835.98 & 835.70 \\
\hline $12 a_{2}$ & 890.16 & 891.74 & 890.65 & 890.26 & 890.55 \\
\hline 13 & 637.36 & 634.38 & 636.01 & 635.92 & 635.70 \\
\hline $14 b_{1}$ & 849.72 & 850.03 & 850.63 & 849.07 & 849.10 \\
\hline 15 & 628.80 & 626.80 & 628.87 & 628.65 & 628.63 \\
\hline$\left|\Delta_{a v}\right|^{a}$ & 1.2 & 2.7 & 1.6 & 0.2 & 0.0 \\
\hline$\left|\Delta_{\max }\right|^{b}$ & 3.2 & 9.8 & 5.9 & 1.2 & 0.0 \\
\hline
\end{tabular}

a) Level of electronic structure theory for calculating the normal coordinates.

b) Mean and maximum absolute deviation with respect to results obtained from cs-VCI calculations based on multi-level potential energy surfaces spanned by CCSD(T) normal coordinates (last column). 
Table VII: Optimized internal coordinates for CHFClI at various levels of theory. Distances $r_{e}$ in Angstrom, bond and torsion angles, $\alpha_{e}$ and $\tau_{e}$, in degrees.

\begin{tabular}{lrrrrrrrr}
\hline \hline & \multicolumn{2}{c}{ MP2 } & & \multicolumn{4}{c}{$\operatorname{CCSD}(\mathrm{T})$} \\
\cline { 2 - 3 } \cline { 6 - 8 } Parameter & avtz & avqz & & avtz & avqz & CBS-3 $^{a}$ & CBS-2 $^{b}$ \\
\hline$r_{e}^{C H}$ & 1.0837 & 1.0832 & & 1.0847 & 1.0843 & 1.0845 & 1.0840 \\
$r_{e}^{C F}$ & 1.3549 & 1.3515 & & 1.3550 & 1.3506 & 1.3482 & 1.3474 \\
$r_{e}^{C C l}$ & 1.7574 & 1.7513 & & 1.7671 & 1.7604 & 1.7563 & 1.7555 \\
$r_{e}^{C I}$ & 2.1379 & 2.1398 & & 2.1490 & 2.1513 & 2.1534 & 2.1529 \\
$\alpha_{e}^{H C I}$ & 107.55 & 107.42 & & 107.62 & 107.42 & & \\
$\alpha_{e}^{F C I}$ & 109.50 & 109.42 & & 109.46 & 109.39 & & \\
$\alpha_{e}^{C l C I}$ & 112.18 & 112.21 & & 112.27 & 112.29 & & \\
$\tau_{e}^{C l C I H}$ & 119.45 & 119.49 & & 119.43 & 119.42 & & \\
$\tau_{e}^{F C I H}$ & -118.80 & -118.71 & & -118.92 & -118.83 & & \\
\hline \hline
\end{tabular}

a) Complete basis set limit using the three-parameter extrapolation formula (CBS-3) of Peterson et al. [31].

b) Complete basis set limit using the two-parameter formula (CBS-2) of Helgaker et al. $[32,33]$. 
Table VIII: Vibrationally averaged structures of CHFCII and CDFClI. Distances, $r_{z}$, in Ångstrom, bond and torsion angles, $\alpha_{z}$ and $\tau_{z}$, in degrees.

\begin{tabular}{lccccr}
\hline \hline & \multicolumn{2}{c}{ CHFClI } & & \multicolumn{2}{c}{ CDFClI } \\
\cline { 2 - 3 } \cline { 5 - 6 } & $\mathrm{MP2}^{a}$ & $\mathrm{CCSD}(\mathrm{T})^{a}$ & & $\mathrm{MP}^{a}$ & $\mathrm{CCSD}(\mathrm{T})^{a}$ \\
\hline$r_{z}^{C H}$ & 1.0930 & 1.0930 & & 1.0908 & 1.0908 \\
$r_{z}^{C F}$ & 1.3603 & 1.3602 & & 1.3602 & 1.3601 \\
$r_{z}^{C C l}$ & 1.7732 & 1.7730 & & 1.7727 & 1.7726 \\
$r_{z}^{C I}$ & 2.1550 & 2.1550 & & 2.1546 & 2.1545 \\
$\alpha_{z}^{H C I}$ & 107.84 & 107.83 & & 107.80 & 107.79 \\
$\alpha_{z}^{F C I}$ & 109.32 & 109.32 & & 109.28 & 109.36 \\
$\alpha_{z}^{C l C I}$ & 112.16 & 112.16 & & 112.20 & 112.20 \\
$\tau_{z}^{C l C I H}$ & 119.67 & 119.66 & & 119.63 & 119.61 \\
$\tau_{z}^{F C I H}$ & -119.01 & -119.01 & -118.95 & -118.96 \\
\hline \hline
\end{tabular}

a) Level of electronic structure theory for calculating the normal coordinates. The wavefunction of the vibrational ground state used for calculating the vibrationally averaged parameters was obtained from a multi-level potential (see text). 
Table IX: Experimental and calculated rotational constants (in $\mathrm{MHz}$ ) for $\mathrm{CH}^{35} \mathrm{FClI}$ and $\mathrm{CH}^{37} \mathrm{FClI}$

\begin{tabular}{|c|c|c|c|c|c|c|c|}
\hline \multirow[b]{2}{*}{ Method } & \multirow[b]{2}{*}{ Type } & \multicolumn{3}{|c|}{$\mathrm{CH}^{35} \mathrm{FClI}$} & \multicolumn{3}{|c|}{$\mathrm{CH}^{37} \mathrm{FClI}$} \\
\hline & & $\mathrm{A}$ & $\mathrm{B}$ & $\mathrm{C}$ & $\mathrm{A}$ & $\mathrm{B}$ & $\mathrm{C}$ \\
\hline Exp. $^{a}$ & Reference & 6278.65 & 1474.15 & 1224.41 & 6192.86 & 1432.70 & 1192.57 \\
\hline $\operatorname{CCSD}(\mathrm{T}) / \mathrm{avtz}$ & Equilibrium & 6248.61 & 1461.53 & 1215.06 & 6163.50 & 1420.27 & 1183.34 \\
\hline $\operatorname{CCSD}(\mathrm{T}) /$ avqz & Equilibrium & 6282.95 & 1462.85 & 1217.11 & 6197.13 & 1421.64 & 1185.37 \\
\hline $\mathrm{CCSD}(\mathrm{T}) / \mathrm{CBS}-3^{b}$ & Equilibrium & 6304.97 & 1462.81 & 1217.84 & 6218.61 & 1421.66 & 1186.12 \\
\hline $\operatorname{CCSD}(\mathrm{T}) / \mathrm{CBS}-2^{c}$ & Equilibrium & 6311.32 & 1463.70 & 1218.66 & 6224.85 & 1422.53 & 1186.92 \\
\hline $\operatorname{CCSD}(\mathrm{T}) / \mathrm{avtz}$ & Vibr. averaged & 6207.64 & 1454.34 & 1209.12 & 6123.60 & 1413.23 & 1177.54 \\
\hline
\end{tabular}

a) Experimental value taken from Ref. [13].

b) Complete basis set limit using the three-parameter extrapolation formula (CBS-3) of Peterson et al. [31].

c) Complete basis set limit using the two-parameter formula (CBS-2) of Helgaker et al. [32, 33]. 
Table X: Harmonic vibrational frequencies $\omega_{i}\left(\right.$ in $\left.\mathrm{cm}^{-1}\right)$ and intensities (in $\mathrm{km} / \mathrm{mol}$ ) for CHFClI.

\begin{tabular}{|c|c|c|c|c|c|}
\hline \multirow[b]{3}{*}{ Mode } & \multicolumn{4}{|c|}{ MP2 } & \multirow{3}{*}{$\begin{array}{r}\operatorname{CCSD}(\mathrm{T}) \\
\text { avtz } \\
\omega\end{array}$} \\
\hline & \multicolumn{2}{|c|}{ avtz } & \multicolumn{2}{|c|}{ avqz } & \\
\hline & $\omega$ & Int. & $\omega$ & Int. & \\
\hline$\omega_{1}$ & 3183.2 & 0.3 & 3182.7 & 0.3 & 3158.9 \\
\hline$\omega_{2}$ & 1332.0 & 9.0 & 1334.1 & 8.7 & 1326.7 \\
\hline$\omega_{3}$ & 1212.5 & 75.0 & 1212.6 & 73.7 & 1202.1 \\
\hline$\omega_{4}$ & 1083.1 & 172.5 & 1084.7 & 173.0 & 1087.6 \\
\hline$\omega_{5}$ & 797.6 & 163.9 & 802.8 & 162.6 & 782.2 \\
\hline$\omega_{6}$ & 615.5 & 32.5 & 610.3 & 35.0 & 602.2 \\
\hline$\omega_{7}$ & 419.6 & 0.4 & 421.5 & 0.4 & 414.0 \\
\hline$\omega_{8}$ & 278.0 & 0.3 & 277.3 & 0.3 & 273.7 \\
\hline$\omega_{9}$ & 197.1 & 0.0 & 197.3 & 0.0 & 193.1 \\
\hline
\end{tabular}


Table XI: Harmonic vibrational frequencies $\omega_{i}\left(\right.$ in $\mathrm{cm}^{-1}$ ) and intensities (in $\mathrm{km} / \mathrm{mol}$ ) for CDFClI.

\begin{tabular}{|c|c|c|c|c|c|}
\hline \multirow[b]{3}{*}{ Mode } & \multicolumn{4}{|c|}{ MP2 } & \multirow{3}{*}{$\begin{array}{r}\operatorname{CCSD}(\mathrm{T}) \\
\mathrm{avtz} \\
\omega\end{array}$} \\
\hline & \multicolumn{2}{|c|}{ avtz } & \multicolumn{2}{|c|}{ avqz } & \\
\hline & $\omega$ & Int. & $\omega$ & Int. & \\
\hline$\omega_{1}$ & 2344.2 & 0.5 & 2343.8 & 0.5 & 2325.9 \\
\hline$\omega_{2}$ & 1086.5 & 170.8 & 1087.7 & 169.7 & 1091.8 \\
\hline$\omega_{3}$ & 985.0 & 8.8 & 987.7 & 9.0 & 980.8 \\
\hline$\omega_{4}$ & 921.2 & 147.0 & 920.3 & 147.9 & 912.9 \\
\hline$\omega_{5}$ & 762.3 & 89.8 & 766.7 & 87.7 & 747.8 \\
\hline$\omega_{6}$ & 572.4 & 21.1 & 568.2 & 22.9 & 559.9 \\
\hline$\omega_{7}$ & 417.5 & 0.4 & 419.4 & 0.5 & 412.0 \\
\hline$\omega_{8}$ & 277.5 & 0.3 & 276.8 & 0.3 & 273.2 \\
\hline$\omega_{9}$ & 196.4 & 0.0 & 196.6 & 0.0 & 192.4 \\
\hline
\end{tabular}


Table XII: Fundamental frequencies $\nu_{i}$ for CHFClI and CDFClI $\left(\right.$ in $\left.\mathrm{cm}^{-1}\right)$.

\begin{tabular}{|c|c|c|c|c|c|}
\hline \multirow[b]{2}{*}{ Mode } & \multicolumn{3}{|c|}{ CHFClI } & \multicolumn{2}{|c|}{ CDFClI } \\
\hline & $\mathrm{MP}^{a}$ & $\operatorname{CCSD}(\mathrm{T})^{a}$ & Exp. ${ }^{b}$ & $\mathrm{MP}^{a}$ & $\operatorname{CCSD}(\mathrm{T})^{a}$ \\
\hline$\nu_{1}$ & 3014.9 & 3014.7 & 3010 & 2255.4 & 2255.1 \\
\hline$\nu_{2}$ & 1296.9 & 1297.4 & 1301 & 1061.4 & 1061.8 \\
\hline$\nu_{3}$ & 1179.4 & 1179.3 & 1180 & 962.6 & 962.3 \\
\hline$\nu_{4}$ & 1059.5 & 1059.6 & 1053 & 895.9 & 895.7 \\
\hline$\nu_{5}$ & 764.6 & 765.4 & 764 & 731.9 & 733.0 \\
\hline$\nu_{6}$ & 594.2 & 594.8 & 587 & 554.2 & 554.2 \\
\hline$\nu_{7}$ & 409.5 & 409.7 & 418 & 407.6 & 407.6 \\
\hline$\nu_{8}$ & 271.0 & 271.2 & 274 & 270.5 & 270.6 \\
\hline$\nu_{9}$ & 191.7 & 191.9 & 197 & 191.1 & 191.1 \\
\hline
\end{tabular}

a) Level of electronic structure theory for calculating the normal coordinates. The wavefunction of the vibrational ground state was obtained from a multi-level potential (see text).

b) Taken from Ref. 13. There is a misprint in Table 3 of Ref. 13. According to private communication with Dr. J. Crassous the given Raman frequency of $3150 \mathrm{~cm}^{-1}$ has to be replaced by $3010 \mathrm{~cm}^{-1}$. 